\title{
Weyl consistency conditions in non-relativistic quantum field theory
}

\section{Sridip Pal and Benjamín Grinstein}

Department of Physics, University of California, San Diego, 9500 Gilman Drive, La Jolla, CA 92093, U.S.A.

E-mail: srpal@ucsd.edu, bgrinstein@ucsd.edu

ABSTRACT: Weyl consistency conditions have been used in unitary relativistic quantum field theory to impose constraints on the renormalization group flow of certain quantities. We classify the Weyl anomalies and their renormalization scheme ambiguities for generic non-relativistic theories in $2+1$ dimensions with anisotropic scaling exponent $z=2$; the extension to other values of $z$ are discussed as well. We give the consistency conditions among these anomalies. As an application we find several candidates for a $C$-theorem. We comment on possible candidates for a $C$-theorem in higher dimensions.

Keywords: Anomalies in Field and String Theories, Conformal and W Symmetry, Renormalization Group

ARXIV EPRINT: 1605.02748 


\section{Contents}

1 Introduction $\quad 1$

2 Generalities $\quad 4$

2.1 Dynamical exponent 8

$3 d=2, z=2$ non relativistic theory 10

$\begin{array}{ll}3.1 \text { Listing out terms } & 10\end{array}$

$\begin{array}{lll}3.2 & \text { Using counter-terms } & 13\end{array}$

$\begin{array}{lll}3.3 & \text { Consistency conditions and vanishing anomalies } & 14\end{array}$

$\begin{array}{lll}3.4 & \text { Applications } & 15\end{array}$

4 Generalisation to arbitrary $z$ value 17

5 A candidate for a $C$-theorem in $d+1 \mathrm{D} \quad 18$

6 Summary and discussion $\quad 22$

A Consistency conditions for $2+1 d$ NRCFT 24

B Anomaly ambiguities $\quad 25$

B.1 $\partial_{t}^{2}$ sector $\quad 26$

B.2 $\partial_{t} \nabla^{2}$ sector 26

$\begin{array}{lll}\text { B.3 } & \nabla^{4} \text { sector } & 27\end{array}$

C S-theorem: $0+1 \mathrm{D}$ conformal quantum mechanics 28

\section{Introduction}

Aspects of the behavior of systems at criticality are accessible through renormalization group (RG) methods. Famously, most critical exponents are determined by a few anomalous dimensions of operators. However, additional information, such as dynamical (or anisotropic) exponents and amplitude relations can be accessed via renormalization group methods near but not strictly at criticality. Far away from critical points there are often other methods, e.g., mean field approximation, that can give more detailed information. The renormalization group used away from critical points can valuably bridge the gap between these regions.

Systems of non-relativistic particles at unitarity, in which the $S$-wave scattering length diverges, $|a| \rightarrow \infty$, exhibit non-relativistic conformal symmetry. Ultracold atom gas experiments have renewed interest in study of such theories. In these experiments one can 
freely tune the $S$-wave scattering length along an RG flow [1, 2]: at $a^{-1}=-\infty$ the system is a BCS superfluid while at $a^{-1}=\infty$ it is a BEC superfluid. The BCS-BEC crossover, at $a^{-1}=0$, is precisely the unitarity limit, exhibiting conformal symmetry. This is a regime where universality is expected, with features independent of any microscopic details of the atomic interactions [3-6]. Other examples of non-relativistic systems with accidentally large scattering cross section include few nucleon systems like the deuteron $[7,8]$ and several atomic systems, including ${ }^{85} \mathrm{Rb}[9],{ }^{138} \mathrm{Cs}[10,11],{ }^{39} \mathrm{~K}[12]$.

In the context of critical dynamics the response function exhibits dynamical scaling. This is characterized by a dynamical scaling exponent which characterizes anisotropic scaling in the time domain. There has been recent interest in anisotropic scaling in systems that are non-covariant extensions of relativistic systems. The ultraviolet divergences in quantized Einstein gravity are softened if the theory is modified by inclusion of higher derivative terms in the Lagrangian. Since time derivatives higher than order 2 lead to the presence of ghosts, ${ }^{1}$ Horava suggested extending Einstein gravity by terms with higher spatial derivatives but only order-2 time derivatives [18]. The mismatch in the number of spatial versus time derivatives is a version of anisotropic scaling, similar to that found in the non-relativistic context. This has motivated studies of extensions of relativistic quantum field theories that exhibit anisotropic scaling at short distances. Independently, motivated by the study of Lorentz violating theories of elementary particle interactions [19], Anselmi found a critical point with exact anisotropic scaling, a so-called Lifshitz fixed point, in his studies of renormalization properties of interacting scalar field theories [20]; see refs. [21, 22] for the case of gauge theories. Anomalous breaking of anisotropic scaling symmetry in the quantum Lifshitz model has been studied in refs. [23-27]; see also ref. [28] for an analysis using holographic methods.

Wess-Zumino consistency conditions for Weyl transformations have been used in unitary relativistic quantum field theory to impose constraints on the renormalization group flow of Weyl anomalies [29]. In $1+1$ dimensions a combination of these anomalies gives Zamolodchikov's $C$-function [30], that famously decreases monotonically along flows towards long distances, is stationary at fixed points and equals the central charge of the 2D conformal field theory at the fixed point boundaries of the flow. Weyl consistency conditions can in fact be used to recover this result [29]. Along the same lines, in $3+1$ dimensions Weyl consistency conditions can be used to show that a quantity $\tilde{a}$ satisfies

$$
\mu \frac{d \tilde{a}}{d \mu}=\mathcal{H}_{\alpha \beta} \beta^{\alpha} \beta^{\beta}
$$

where $\mu$ is the renormalization group scale, increasing towards short distances. The equation shows that at fixed points, characterized by $\mu d g^{\alpha} / d \mu \equiv \beta^{\alpha}=0, \tilde{a}$ is stationary. It can be shown in perturbation theory that $\mathcal{H}_{\alpha \beta}$ is a positive definite symmetric matrix [31]. By construction the quantity $\tilde{a}$ is, at fixed points, the conformal anomaly $a$ of Cardy, associ-

\footnotetext{
${ }^{1}$ Generically, the $S$-matrix in models with ghosts is not unitary. However, under certain conditions on the spectrum of ghosts and the nature of their interactions, a unitary $S$-matrix is possible [13-16]. In theories of gravity Hawking and Hertog have proposed that ghosts lead to unitarity violation at short distances, and unitarity is a long-distance emergent phenomenon [17].
} 
ated with the Euler density conformal anomaly when the theory is placed in a curved background [32]. This is then a 4-dimensional generalization of Zamolodchikov's $C$ function, at least in perturbation theory. Going beyond 4 dimensions, Weyl consistency conditions can be used to show that in $d=2 n$ dimensions there is a natural quantity that satisfies (1.1), and that this quantity is at fixed points the anomaly associated with the $d$-dimensional Euler density [33]. Concerns about the viability of a $C$-theorem in 6-dimensions were raised by explicit computations of "metric" $\mathcal{H}_{\alpha \beta}$ in perturbation theory [34-36]. However it was discovered in ref. [37] that there exists a one parameter family of extensions of the the quantity $\tilde{a}$ of ref. [33] that obey a $C$-theorem perturbatively.

Weyl consistency conditions can also be used to constrain anomalies in non-relativistic field theories. The constraints imposed at fixed points have been studied in ref. [23] for models with anisotropic scaling exponent $z=2$ in 2-spatial dimensions; see refs. [38, 39] for studies of the Weyl anomaly at $d=4, z=3$ and $d=6$. Here we investigate constraints imposed along renormalization group flows. We recover the results of [23] by approaching the critical points along the flows. As mentioned above, there are questions that can only be accessed through the renormalization group methods applied to flows, away from fixed points. The additional information obtained from consideration of Weyl consistency conditions on flows can be used to ask a number of questions. For example, we may ask if there is a suitable candidate for a $C$-theorem.

A related issue is the possibility of recursive renormalization group flows. Recursive flows in the perturbative regime have been found in several examples in $4-\epsilon$ and in 4 dimensional relativistic quantum field theory [40-45]. Since Weyl consistency conditions imply $\tilde{a}$ does not increase along RG-flows it must be that $\tilde{a}$ remains constant along recursive flows. This can be shown directly, that is, without reference to the monotonicity of the flow; see [45]. In fact one can show that on recursive flows all physical quantities, not just $\tilde{a}$, remain constant: the recursive flow behaves exactly the same as a single fixed point. This is as it should be: the monotonicity of the flow of $a$ implies that limit cycles do not exist in any physically meaningful sense [46, 47]; in fact, they may be removed by a field and coupling constant redefinition. However, it is well known that bona-fide renormalization group limit cycles exist in some non-relativistic theories [48-50]. The $C$-theorem runs afoul of limit-cycles, and an immediate question then is what invalidates it in models that exhibit recursive flows? Our analysis indicates some potential candidates for $C$-theorems but does not show whether generically the "metric" $\mathcal{H}_{\alpha \beta}$ has definite sign. The question of under what conditions the metric has definite sign, precluding recursive flows, is left open for further investigation.

The paper is organized as follows. In section 2 we set-up the computation, using a background metric and space and time dependent coupling constants that act as sources of marginal operators. In the section we also clarify the relation between the dynamical exponent and the classical anisotropic exponent. We then use this formalism in section 3 where we analyze the consistency conditions for the case of 2-spatial dimensions and anisotropic exponent $z=2$. The Weyl consistency conditions and scheme dependent ambiguities are lengthy, so they are collected in appendices A and B. In section 4 we explore the case of arbitrary $z$, extending some of the results of the previous section and in section 5 we 
propose a candidate $C$-theorem for any even spatial dimension. We offer some general conclusions and review our results in section 6 . There is no trace anomaly equation for the case of zero spatial derivatives, that is, particle quantum mechanics; we comment on this, and present a simple but useful theorem that does apply in this case, in the final appendix, appendix C.

\section{Generalities}

We consider non-relativistic (NR) field theories with point-like interactions. Although not necessary for the computation of Weyl consistency conditions, it is convenient to keep in mind a Lagrangian description of the model. The Lagrangian density $\mathcal{L}=\mathcal{L}(\phi, m, g)$ is a function of fields $\phi(t, \vec{x})$, mass parameters $m$ and coupling constants $g$ that parametrize interaction strengths. We restrict our attention to models for which the action integral,

$$
S[\phi(\vec{x}, t)]=\int d t d^{d} x \mathcal{L}
$$

remains invariant under the rescaling

$$
\vec{x} \mapsto \lambda \vec{x}, \quad t \mapsto \lambda^{z} t,
$$

that is,

$$
S\left[\lambda^{\Delta} \phi\left(\lambda \vec{x}, \lambda^{z} t\right)\right]=S[\phi(\vec{x}, t)] .
$$

Here $\Delta$ is the matrix of canonical dimensions of the fields $\phi$. In a multi-field model the anisotropic scaling exponent $z$ is common to all fields. Moreover, assuming that the kinetic term in $\mathcal{L}$ is local, so that it entails powers of derivative operators, $z$ counts the mismatch in the number of time derivatives and spatial derivatives. In the most common cases there is a single time derivative and $z$ spatial derivatives so that $z$ is an integer.

For a simple example, useful to keep in mind for orientation, the action for a single complex scalar field with anisotropic scaling $z$ in $d$ dimensions is given by

$$
S=\int d t d^{d} x\left[i m \phi^{*} \overleftrightarrow{\partial_{t}} \phi-\vec{\nabla}_{i_{1}} \cdots \vec{\nabla}_{i_{z / 2}} \phi^{*} \vec{\nabla}_{i_{1}} \cdots \vec{\nabla}_{i_{z / 2}} \phi-g m^{z / d}|\phi|^{2 N}\right]
$$

where $z$ is an even integer so that the Lagrangian density is local. If $N=1+z / d$ the scaling property (2.1) holds with $\Delta=d / 2$ (alternatively, if $N \in \mathbb{Z}$, then $z=d(N-1) \in d \mathbb{Z}$ ). When (2.1) holds the coupling constant $g$ is dimensionless. The mass parameters $m$ have dimensions of $T / L^{z}$, where $T$ and $L$ are time and space dimensions, respectively. One may use the mass parameter to measure time in units of $z$-powers of length, and this can be implemented by absorbing $m$ into a redefinition, $t=m \hat{t}$. In multi-field models one can arbitrarily choose one of the masses to give the conversion factor and then the independent mass ratios are dimensionless parameters of the model. In models that satisfy the scaling property (2.1), these mass ratios together with the coefficients of interaction terms comprise the set of dimensionless couplings that we denote by $g^{\alpha}$ below.

The above setup is appropriate for studies of, say, quantum criticality. However the calculations we present are applicable to studies of thermal systems in equilibrium since 
the imaginary time version of the action integral is equivalent to an energy functional in $d+1$ spatial dimensions. Taking $t=-i y$ in the example of eq. (2.2) the corresponding energy integral is

$$
H=\int d y d^{d} x\left[m \phi^{*} \overleftrightarrow{\partial_{y}} \phi+\vec{\nabla}_{i_{1}} \cdots \vec{\nabla}_{i_{z / 2}} \phi^{*} \vec{\nabla}_{i_{1}} \cdots \vec{\nabla}_{i_{z / 2}} \phi+g m^{z / d}|\phi|^{2 N}\right] .
$$

The short distance divergences encountered in these models need to be regularized and renormalized. Although our results do not depend explicitly on the regulator used, it is useful to keep in mind a method like dimensional regularization that retains most symmetries explicitly. Thus we consider NR field theories in $1+n$ dimensions, where the spatial dimension $n=d-\epsilon$, with $d$ an integer. Dimensional regularization requires the introduction of a parameter $\mu$ with dimensions of inverse length, $L^{-1}$. Invariance under (2.1) is then broken, but can be formally recovered by also scaling $\mu$ appropriately, $\mu \mapsto \lambda^{-1} \mu$. For an example, consider the dimensionally regularized version of (2.2):

$$
S\left[\phi_{0}(\vec{x}, t) ; \mu\right]=\int d t d^{n} x\left[i m_{0} \phi_{0}^{*} \overleftrightarrow{\partial_{t}} \phi_{0}-\vec{\nabla}_{i_{1}} \cdots \vec{\nabla}_{i_{z / 2}} \phi_{0}^{*} \vec{\nabla}_{i_{1}} \cdots \vec{\nabla}_{i_{z / 2}} \phi_{0}-g Z_{g} m_{0}^{z / d} \mu^{k \epsilon}\left|\phi_{0}\right|^{2 N}\right] \text {. }
$$

We have written this in terms of bare field and mass, $\phi_{0}$ and $m_{0}$, and have given the bare coupling constant explicitly in terms of the renormalized one, $g_{0}=\mu^{k \epsilon} Z_{g} g$. The coefficient $k=N-1=z / d$ is dictated by dimensional analysis. It follows that

$$
S\left[\lambda^{n / 2} \phi_{0}\left(\lambda \vec{x}, \lambda^{z} t\right) ; \lambda^{-1} \mu\right]=S\left[\phi_{0}(\vec{x}, t) ; \mu\right]
$$

In order to study the response of the system to sources that couple to the operators in the interaction terms of the Lagrangian, we generalize the coupling constants $g^{\alpha}$ to functions of space and time $g^{\alpha}(t, \vec{x})$. One can then obtain correlation functions of these operators by taking functional derivatives of the partition function with respect to the space-time dependent couplings, and then setting the coupling functions to constant values. Additional operators of interest are obtained by placing these systems on a curved background, with metric $\gamma_{\mu \nu}(t, \vec{x})$. One can then obtain correlations including components of the stress-energy tensor by taking functional derivatives with respect to the metric and evaluating these on a trivial, constant metric. For example, we then can define the components of the symmetric quantum stress energy tensor and finite composite operators in the following way:

$$
T_{\mu \nu}=\frac{2}{\sqrt{\gamma}} \frac{\delta S_{0}}{\delta \gamma^{\mu \nu}} \quad\left[\mathcal{O}_{\alpha}\right]=\frac{1}{\sqrt{\gamma}} \frac{\delta S_{0}}{\delta g^{\alpha}}
$$

The square bracket notation in the last term indicates that these are finite operators, possibly differing from $\mathcal{O}_{\alpha}=\partial \mathcal{L} / \partial g^{\alpha}$ by a total derivative term.

Time plays a special role in theories with anisotropic scaling symmetry. Hence, it is useful to assume the background space-time, in addition to being a differential manifold $\mathcal{M}$, carries an extra structure - we can foliate the space-time with a foliation of co-dimension 1. This can be thought of a topological structure on $\mathcal{M}$ [18], before any notion of Riemannian metric is introduced on such manifold. Now the co-ordinate transformations that preserve the foliation are of the form:

$$
t \mapsto \tau(t), x^{i} \mapsto \xi^{i}(\vec{x}, t)
$$


We will also assume the space-time foliation is topologically given by $\mathcal{M}=R \times \Sigma$. The foliation can be given Riemannian structure with three basic objects: $h_{i j}, N_{i}$ and $N$. This is the ADM decomposition of the metric - one can generally think as writing the metric in terms of lapse and shift functions, $N(t, \vec{x})$ and $N_{i}(t, \vec{x})$, and a metric on spatial sections, $h_{i j}(t, \vec{x})$ :

$$
d s^{2}=\gamma_{\mu \nu} d x^{\mu} d x^{\nu}=N^{2} d t^{2}+2 N_{i} d t d x^{i}-h_{i j} d x^{i} d x^{j}
$$

Here and below the latin indices run over spatial coordinates, $i, j=1, \ldots, d$. We assume invariance of the theory under foliation preserving diffeomorphisms. In a non-relativistic set up, it is convenient to remove the shift $N^{i}$ by a foliation preserving map $t \mapsto \tau(t)$ and $x^{i} \mapsto \xi^{i}(\vec{x}, t)$. The metric is then given by

$$
d s^{2}=\gamma_{\mu \nu} d x^{\mu} d x^{\nu}=N^{2} d t^{2}-h_{i j} d x^{i} d x^{j}
$$

Once the shift functions are removed the restricted set of diffeomorphisms that do not mix space and time are allowed, $t \rightarrow \tau(t)$ and $x^{i} \rightarrow \xi^{i}(x)$, so that $N^{i}=0$ is preserved.

In Euclidean space, the generating functional of connected Green's functions $W$ is given by

$$
e^{W}=\int[d \phi] e^{-S_{0}-\Delta S}
$$

The action integral for these models is generically of the form

$$
S_{0}=\int d t d^{n} x N \sqrt{h} \mathcal{L}_{0}
$$

where $h=\operatorname{det}\left(h_{i j}\right)$. We have denoted by $\mathcal{L}_{0}$ the Lagrangian density with bare fields and couplings as arguments; these are to be expressed in terms of the renormalized fields and couplings, so as to render the functional integral finite. The term $\Delta S$ contains additional counter-terms that are solely functionals of $g^{\alpha}$ and $\gamma_{\mu \nu}$ that are also required in order to render $W$ finite. In a curved background the scaling (2.1) can be rephrased in terms of a transformation of the metric,

$$
N(\vec{x}, t) \mapsto \lambda^{z} N(\vec{x}, t), \quad h_{i j}(\vec{x}, t) \mapsto \lambda^{2} h_{i j}(\vec{x}, t)
$$

Then the generalization of the formal invariance of eq. (2.4) is

$$
S_{0}\left[\lambda^{z} N(\vec{x}, t), \lambda^{2} h_{i j}(\vec{x}, t), \lambda^{\Delta_{0}} \phi_{0}(\vec{x}, t) ; \lambda^{-1} \mu\right]=S_{0}\left[N(\vec{x}, t), h_{i j}(\vec{x}, t), \phi_{0}(\vec{x}, t) ; \mu\right]
$$

for a suitable matrix of canonical dimensions $\Delta_{0}$ of the bare fields (appropriate to $n=d-\epsilon$ spatial dimensions).

We assume that when introducing a curved background the action integral is suitably modified so that the formal symmetry of eq. (2.12) holds locally, that is, it holds when replacing $\lambda \rightarrow \exp (-\sigma(\vec{x}, t))$. The modification to the action integral consists of additional terms that couple the fields $\phi$ to the background curvature. 
For example, the model in eq. (2.3) for $z=2$ is modified to include, in addition to coupling to a background metric, additional terms

$$
\begin{gathered}
\int d t d^{n} x N \sqrt{h}\left[i m_{0} \xi_{K} \phi_{0}^{*} \phi_{0} K+\xi_{N \phi}\left(\phi_{0}^{*} \frac{\partial_{i} N}{N} \partial^{i} \phi_{0}+\phi_{0} \frac{\partial_{i} N}{N} \partial^{i} \phi_{0}^{*}\right)\right. \\
\left.+\xi_{N N} \frac{\partial_{i} N}{N} \frac{\partial^{i} N}{N} \phi_{0}^{*} \phi_{0}+\xi_{R} R \phi_{0}^{*} \phi_{0}\right] .
\end{gathered}
$$

Here $K_{i j}=\frac{1}{2} \partial_{t} h_{i j} / N$ is the extrinsic curvature of the $t=$ constant hypersurfaces in the $N^{i}=0$ gauge and $K=h^{i j} K_{i j}$ (with $h^{i j}$ the inverse of the metric $h_{i j}$ ), and $R$ is the $d$-dimensional Ricci scalar for the metric $h_{i j}$. Under the transformation (2.11) with $\lambda=$ $\exp (-\sigma)$ one has $K \rightarrow e^{2 \sigma}\left(K+n \partial_{t} \sigma / N\right), R \rightarrow e^{2 \sigma}\left(R+2(n-1) \nabla^{2} \sigma-(n-1)(n-2) \nabla_{i} \sigma \nabla^{i} \sigma\right)$ and $N \rightarrow e^{-2 \sigma} N$, so that choosing $\xi_{K}=1 / 2$ and ensuring

$$
2(n-1) \xi_{R}+2 \xi_{N \phi}+\frac{n}{2}=0 \quad(n+2) \xi_{N \phi}-4 \xi_{N N}+\frac{n}{2}=0,
$$

the action integral remains invariant. Thus, we have a one parameter family of parameters that preserves invariance of the action under anisotropic scaling. For arbitrary even $z$ and arbitrary spatial dimension $n$, in the example (2.3) we first integrate by parts the spatial covariant derivatives:

$$
\vec{\nabla}_{i_{1}} \cdots \vec{\nabla}_{i_{z / 2}} \phi^{*} \vec{\nabla}_{i_{1}} \cdots \vec{\nabla}_{i_{z / 2}} \phi \rightarrow(-1)^{z / 2} \phi^{*}\left(\nabla^{2}\right)^{z / 2} \phi
$$

Then we replace the operator $\left(\nabla^{2}\right)^{\frac{z}{2}}$ by $\mathcal{O}^{(n+2 z-4)} \mathcal{O}^{(n+2 z-8)} \cdots \mathcal{O}^{(n+4)} \mathcal{O}^{(n)}$ with $\mathcal{O}^{(p)}$ defined as

$$
\mathcal{O}^{(p)} \equiv\left[\nabla^{2}-\frac{p}{4(n-1)} R+\frac{2+p-n}{z} \frac{\partial_{i} N}{N} h^{i j} \partial_{j}+\frac{n}{4 z^{2}}(2+p-n) \frac{\partial_{i} N}{N} h^{i j} \frac{\partial_{j} N}{N}\right]
$$

Under $h_{i j} \rightarrow e^{-2 \sigma} h_{i j}, N \rightarrow e^{-z \sigma} N$ and $\psi \rightarrow e^{\frac{p}{2} \sigma} \psi$, this operator transform covariantly, in the sense that

$$
\mathcal{O}^{(p)} \psi \rightarrow e^{\left(\frac{p}{2}+2\right) \sigma} \mathcal{O}^{(p)} \psi
$$

Hence, under the Weyl rescaling $h_{i j} \rightarrow e^{-2 \sigma} h_{i j}, N \rightarrow e^{-z \sigma} N$ and $\phi \rightarrow e^{\frac{n}{2} \sigma} \phi$ we have following, transforming covariantly

$$
\phi_{0}^{*} \mathcal{O}^{(n+2 z-4)} \mathcal{O}^{(n+2 z-8)} \cdots \mathcal{O}^{(n+4)} \mathcal{O}^{(n)} \phi_{0} \rightarrow e^{(n+z) \sigma} \phi_{0}^{*} \mathcal{O}^{(n+2 z-4)} \mathcal{O}^{(n+2 z-8)} \cdots \mathcal{O}^{(n+4)} \mathcal{O}^{(n)} \phi_{0}
$$

For $z=2$, this construction gives

$$
\begin{aligned}
N \sqrt{h} \phi_{0}^{*} \mathcal{O}^{(n)} \phi_{0} & =N \sqrt{h} \phi_{0}^{*}\left[\nabla^{2}-\frac{n}{4(n-1)} R+\frac{\partial_{i} N}{N} h^{i j} \partial_{j}+\frac{n}{8} \frac{\partial_{i} N}{N} h^{i j} \frac{\partial_{j} N}{N}\right] \phi_{0} \\
& =N \sqrt{h}\left[-\partial_{i} \phi_{0}^{*} \partial^{i} \phi_{0}-\frac{n}{4(n-1)} R \phi_{0}^{*} \phi_{0}+\frac{n}{8} \frac{\partial_{i} N}{N} h^{i j} \frac{\partial_{j} N}{N} \phi_{0}^{*} \phi_{0}\right]
\end{aligned}
$$


This solves eq. (2.13) with

$$
\xi_{R}=-\frac{n}{4(n-1)}, \quad \xi_{N \phi}=0, \quad \xi_{N N}=\frac{n}{8} .
$$

The extra freedom for $z=2$ arises from the fact that $\phi_{0}^{*}\left[R+(n-1) \frac{\nabla^{2} N}{N}\right.$ $\left.-\frac{(n-1)(n+2)}{4} \frac{\partial_{i} N}{N} \frac{\partial^{i} N}{N}\right] \phi_{0}$ is Weyl invariant. This special invariant quantity is available only for $z=2$.

Having constructed a classically Weyl invariant curved space action, we have that $\tilde{W}=W-W_{\text {c.t. }}=W+\Delta S$ is invariant under these local transformations:

$$
\tilde{W}\left[e^{-z \sigma} N, e^{-2 \sigma} h_{i j}, g^{\alpha}\left(e^{-\sigma} \mu\right)\right]=\tilde{W}\left[N, h_{i j}, g^{\alpha}(\mu)\right]
$$

We have suppressed the explicit dependence on space and time and have assumed the only dependence on the renormalization scale $\mu$ is implicitly through the couplings: using $\mu$-independence of bare couplings, $g_{0}=\mu^{k \epsilon} g(\mu) Z_{g}(g(\mu))=(\lambda \mu)^{k \epsilon} g(\lambda \mu) Z_{g}(g(\lambda \mu))$ so that $\left(\lambda^{-1} \mu\right)^{k \epsilon} g(\mu) Z_{g}(g(\mu))=\mu^{k \epsilon} g(\lambda \mu) Z_{g}(g(\lambda \mu))$.

The generating functional $W$ is not invariant in the sense of eq. (2.20). The anomalous variation of $W$ arises purely from the counter-terms: under an infinitesimal transformation,

$$
\begin{aligned}
\Delta_{\sigma} W & =W_{\text {c.t. }}\left[(1-z \sigma) N,(1-2 \sigma) h_{i j}, g^{\alpha}-\sigma \mu d g^{\alpha} / d \mu\right]-W_{\text {c.t. }}\left[N, h_{i j}, g^{\alpha}\right] \\
& \left.=\int d t d^{d} x N \sqrt{h} \text { (terms with derivatives on } N, h_{i j}, g^{\alpha} \text { and } \sigma\right)
\end{aligned}
$$

does not vanish. Using eqs. (2.5) and choosing $\sigma$ to be an infinitesimal local test function, this reads

$$
z\left\langle T^{0}{ }_{0}\right\rangle+\left\langle T^{i}{ }_{i}\right\rangle-\beta^{\alpha}\left\langle\left[\mathcal{O}_{\alpha}\right]\right\rangle=\left(\text { terms with derivatives on } N, h_{i j}, g^{\alpha} \text { and } \sigma\right) .
$$

Evaluating at space and time independent coupling constants and on a flat metric, so that the right hand side vanishes, we recognize this as the trace anomaly for NRQFT.

Since the Weyl group is Abelian, consistency conditions follow from requiring that

$$
\left[\Delta_{\sigma}, \Delta_{\sigma^{\prime}}\right] W=0 .
$$

These consistency conditions impose relations on the various anomaly terms on the right hand side of eq. (2.21). In the following sections we classify all possible anomaly terms and derive the relations imposed by these conditions.

\subsection{Dynamical exponent}

In the theory of critical phenomena the dynamical exponent $\zeta$ characterizes how a correlation length scales with time in time dependent correlations. At the classical level (the gaussian fixed point) this just corresponds to the anisotropic exponent $z$ introduced above. To understand the connection between these we must retain explicitly the dependence on the mass parameter(s) $m$ in eqs. (2.12) and (2.20). We consider for simplicity the case of a single mass parameter. In particular, we have

$$
\tilde{W}\left[e^{-z \sigma} N, e^{-2 \sigma} h_{i j}, g^{\alpha}\left(e^{-\sigma} \mu\right), m\left(e^{-\sigma} \mu\right)\right]=\tilde{W}\left[N, h_{i j}, g^{\alpha}(\mu), m(\mu)\right] .
$$


By dimensional analysis and translational and rotational invariance, the correlator of fundamental fields is given by

$$
\langle\phi(\vec{x}, t) \phi(0,0)\rangle=\frac{1}{|\vec{x}|^{2 \Delta}} F\left(\ln \left(m(\mu)|\vec{x}|^{z} / t\right), \ln (\mu|\vec{x}|)\right),
$$

for some dimensionless function of two arguments, $F(x, y)$. This function is further constrained by the renormalization group equation. At a fixed point, $\beta^{\alpha}=0$, it takes the form

$$
\left(\mu \frac{\partial}{\partial \mu}+\gamma_{m} m \frac{\partial}{\partial m}+2 \gamma\right)\langle\phi(\vec{x}, t) \phi(0,0)\rangle=0
$$

where $\gamma_{m}$ and $\gamma$ are the mass anomalous dimension and the field anomalous dimension, respectively. These are generally dimensionless functions of the dimensionless coupling constants, $g^{\alpha}$, here evaluated at their fixed point values, say, $g_{*}^{\alpha}$. It follows that

$$
\langle\phi(\vec{x}, t) \phi(0,0)\rangle=\frac{1}{\mu_{0}^{2 \gamma}|\vec{x}|^{2(\Delta+\gamma)}} f\left(m\left(\mu_{0}\right) \mu_{0}^{-\gamma_{m}}|\vec{x}|^{z-\gamma_{m}} / t\right) .
$$

Here $\mu_{0}$ is a reference renormalization point and $f$ is a dimensionless function of one variable. This shows that at the fixed point the fields scale with dimension $\Delta+\gamma$ and the dynamical exponent is $\zeta=z-\gamma_{m}$. It is important to understand that while $\zeta$ can be thought of as running along flows, the exponent $z$ is fixed to its classical (gaussian fixed point) value.

As an example consider the following Lagrangian for a $z=2$ theory in $4+1$ dimensions:

$$
\mathcal{L}=\left[i Z_{m} m Z_{\phi} \phi^{*} \overleftrightarrow{\partial_{t}} \phi-Z_{\phi} \vec{\nabla} \phi^{*} \vec{\nabla} \phi-\frac{1}{2} Z_{g} g \mu^{\frac{\epsilon}{2}} \sqrt{Z_{m} m} Z_{\phi}^{3 / 2}|\phi|^{2}\left(\phi+\phi^{*}\right)\right]
$$

The renormalization factors in dimensional regularization in $n+1$ dimensions, with $n=$ $4-\epsilon$, have the following form:

$$
Z_{X}=1+\sum_{n=1} \frac{a_{n}^{X}}{\epsilon^{n}},
$$

where the residues $a_{n}^{X}$ are functions of the renormalized coupling constant $g$. Independence of the bare parameters on the scale $\mu$ requires

$$
0=\mu \frac{d}{d \mu}\left(Z_{g} g \mu^{\frac{\epsilon}{2}}\right)=\frac{\partial Z_{g}}{\partial g} \hat{\beta} g \mu^{\frac{\epsilon}{2}}+Z_{g} \hat{\beta} \mu^{\frac{\epsilon}{2}}+\frac{\epsilon}{2} Z_{g} g \mu^{\frac{\epsilon}{2}}
$$

where $\hat{\beta} \equiv \mu d g / d \mu$ has $\hat{\beta}(g, \epsilon)=-\frac{\epsilon}{2} g+\beta(g)$, and

$$
0=\mu \frac{d}{d \mu}\left(Z_{m} m\right)=\frac{\partial Z_{m}}{\partial g} \hat{\beta} m+\mu \frac{d m}{d \mu} Z_{m} .
$$

It follows that

$$
\gamma_{m}=\mu \frac{d \ln (m)}{d \mu}=\frac{1}{2} g \frac{d a_{1}^{m}}{d g} .
$$

At one loop the self-energy correction to the propagator, represented by the Feynman diagram in figure 1 , reads

$$
i \Sigma(E, \vec{k})=-\frac{1}{2} m g^{2} \mu^{\epsilon} \int \frac{d p_{0}}{2 \pi} \frac{d^{n} p}{(2 \pi)^{n}} D\left(\frac{1}{2} E-p_{0}, \frac{1}{2} \vec{k}-\vec{p}\right) D\left(\frac{1}{2} E+p_{0}, \frac{1}{2} \vec{k}+\vec{p}\right)
$$




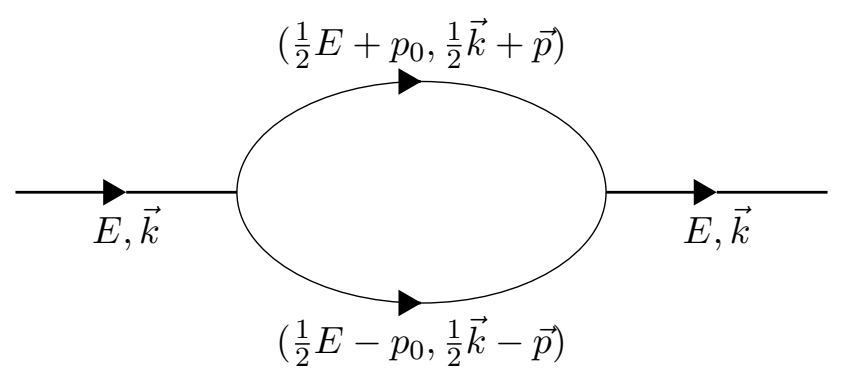

Figure 1. Self energy correction to propagator at one loop.

where the propagator is given by

$$
D(E, \vec{p})=\frac{i}{\left(2 m E-\vec{p}^{2}+i 0^{+}\right)} .
$$

The integration over $p_{0}$ and then over $\vec{p}$ gives

$$
\Sigma(E, \vec{k})=\frac{1}{8} g^{2} \mu^{\epsilon} \int \frac{d^{n} p}{(2 \pi)^{n}} \frac{1}{\left(m E-\frac{1}{4} \vec{k}^{2}-\vec{p}^{2}\right)}=-\frac{1}{\epsilon} \frac{g^{2}}{64 \pi^{2}}\left(m E-\frac{1}{4} \vec{k}^{2}\right)+\cdots,
$$

where the ellipses stand for finite terms. We read off

$$
Z_{\phi}-1=\frac{g^{2}}{256 \pi^{2} \epsilon} \quad \text { and } \quad Z_{m}-1=\frac{g^{2}}{256 \pi^{2} \epsilon}
$$

Form which it follows that

$$
\gamma_{m}=\frac{g^{2}}{256 \pi^{2}}
$$

\section{$3 d=2, z=2$ non relativistic theory}

\subsection{Listing out terms}

We first consider $2+1$ NRCFT with $z=2$. It is convenient to catalogue the possible terms on the right hand side of eq. (2.21) by the number of space and time derivatives acting on the metric, the couplings and the transformation parameter $\sigma$. Rotational invariance implies that space derivatives always appear in contracted pairs. We must, in addition, insure the correct dimensions. Table 1 summarizes the dimensions of the basic rotationally invariant quantities; $R$ stands for the curvature scalar constructed from the spatial metric $h_{i j}$. Since $h_{i j}$ is the metric of a 2 dimensional space, rotational invariants constructed from the Riemann and Ricci tensors can be expressed in terms of $R$ only.

In order to match up the dimension of the Lagrangian, terms that only contain spatial derivatives must have exactly four derivatives. The derivatives can act on the metric or on on the dimensionless variation parameter $\sigma$. Hence we have following 2-spatial-derivatives 


\begin{tabular}{|c|c|c|c|}
\hline Operators & $N$ & $g^{\alpha}$ & $R$ \\
\hline Length Dimension & 0 & 0 & 2 \\
\hline Time Dimension & 1 & 0 & 0 \\
\hline
\end{tabular}

Table 1. Basic rotationally invariant operators and their dimensions.

components:

$$
\begin{gathered}
\frac{\partial_{i} N}{N} \frac{\partial^{i} N}{N}, \quad \frac{\partial_{i} N}{N} \partial^{i} g^{\alpha}, \quad \partial_{i} g^{\alpha} \partial^{i} g^{\beta}, \quad \frac{\nabla^{2} N}{N}, \quad \nabla^{2} g^{\alpha}, \quad R \\
\nabla^{2} \sigma \\
\partial_{i} \sigma \frac{\partial^{i} N}{N}, \quad \partial_{i} \sigma \partial^{i} g^{\alpha}
\end{gathered}
$$

where we note that in the term $\frac{\partial_{i} N}{N}$ the denominator serves to cancel off the time dimension of the numerator. To form a 4 derivative term out of above terms, we can (i) choose two terms among (3.1) with repetition allowed: there are $6^{2}-{ }^{6} C_{2}=21$ such terms; (ii) (3.2) can combine with any of (3.1) giving 6 additional terms; and (iii) we can choose one of (3.3) and choose another from (3.1), yielding an additional $2 * 6=12$ terms. Hence we will have $21+12+6=39$ terms with four space derivatives. Terms with derivatives of $R$, such as

$$
\partial_{i} R \partial^{i} g^{\alpha} \quad \text { and } \quad \partial_{i} R \frac{\partial^{i} N}{N},
$$

are not independent. Integrating by parts, the term $\partial_{i} R \partial^{i} g^{\alpha}$ can written in terms of $R \nabla^{2} g^{\alpha}$ and $R \partial_{i} \sigma \partial^{i} g^{\alpha}$, and the term $R \nabla^{2} N$ can be expressed in terms of $\partial_{i} R \frac{\partial^{i} N}{N}$. The 39 four derivative terms, which we call the $\nabla^{4}$ sector, appear on the right hand side of (2.21) with dimensionless coefficients that are functions of the couplings $g^{\alpha}$, and with a factor of $\sigma$ if the term does not already contain one. Table 2 gives our notation for the coefficients of these terms in eq. (2.21).

Two time derivatives are required for the sector with pure time derivatives, which we label $\partial_{t}^{2}$. The terms must still have length dimension -4 . The dimensions of the basic building blocks are given in table 3 , where $K_{i j}=\frac{1}{2} \partial_{t} h_{i j} / N$ is the extrinsic curvature of the $t=$ constant hypersurfaces in the $N^{i}=0$ gauge and $K=h^{i j} K_{i j}$ (with $h^{i j}$ the inverse of the metric $\left.h_{i j}\right)$. The combination $\left(K_{i j}-\frac{1}{2} K h_{i j}\right)$ is convenient because it is Weyl invariant. Hence, for the $\partial_{t}^{2}$ sector we have the following basic one derivative terms:

$$
\begin{gathered}
K, \quad \partial_{t} g^{\alpha} \\
\partial_{t} \sigma \\
K_{i j}-\frac{1}{2} K h_{i j}
\end{gathered}
$$

The term $\partial_{t} N$ is not included in the list because it is not covariant. The diffeomorphism invariant quantity is given by $\partial_{t} N-\Gamma_{00}^{0} N$ which vanishes identically 0 .

Possible anomaly terms are constructed from the $2^{2}-1=3$ products of terms in (3.4); from 2 terms by combining (3.5) and one from (3.4); and we can have (3.6) contracted 


\begin{tabular}{|c|c|c|c|c|c|c|c|c|c|}
\hline$\nabla^{4}$ Sector & $\partial_{i} N \partial^{i} N$ & $\partial_{i} g^{\alpha} \partial^{i} g^{\beta}$ & $\partial_{i} N \partial^{i} g^{\alpha}$ & $\nabla^{2} N$ & $\nabla^{2} g^{\alpha}$ & $R$ & $\nabla^{2} \sigma$ & $\partial_{i} \sigma \partial^{i} N$ & $\partial_{i} \sigma \partial^{i} g^{\alpha}$ \\
\hline$\partial_{i} N \partial^{i} N$ & $P_{3}, p_{3}$ & $X_{\alpha \beta}, x_{\alpha \beta}$ & $P_{1 \alpha}, \rho_{9 \alpha}$ & $P_{4}, p_{4}$ & $Y_{\alpha}, y_{\alpha}$ & $Q, \chi_{4}$ & $\chi_{3}$ & $\rho_{11}$ & $\rho_{8 \alpha}$ \\
\hline$\partial_{i} g^{\alpha} \partial^{i} g^{\beta}$ & $X_{\alpha \beta}, x_{\alpha \beta}$ & $X_{\alpha \beta \gamma \delta}, x_{\alpha \beta \gamma \delta}$ & $X_{\alpha \beta \gamma}, x_{\alpha \beta \gamma}$ & $X_{2 \alpha \beta}, x_{2 \alpha \beta}$ & $T_{2 \alpha \beta \gamma}, t_{2 \alpha \beta \gamma}$ & $Y_{5 \alpha \beta}, y_{5 \alpha \beta}$ & $a_{3 \alpha \beta}$ & $\rho_{1 \alpha \beta}$ & $t_{\alpha \beta \gamma}$ \\
\hline$\partial_{i} N \partial^{i} g^{\alpha}$ & $P_{1 \alpha}, \rho_{9 \alpha}$ & $X_{\alpha \beta \gamma}, x_{\alpha \beta \gamma}$ & $P_{5 \alpha \beta}, p_{5 \alpha \beta}$ & $P_{25 \alpha}, \rho_{25 \alpha}$ & $P_{26 \alpha \beta}, \rho_{26 \alpha \beta}$ & $\chi_{\alpha}$ & $\chi_{1 \alpha}$ & $\rho_{10 \alpha}$ & $x_{1 \alpha \beta}$ \\
\hline$\nabla^{2} N$ & $P_{4}, p_{4}$ & $X_{2 \alpha \beta}, x_{2 \alpha \beta}$ & $P_{25 \alpha}, \rho_{25 \alpha}$ & $P_{23}, \rho_{23}$ & $P_{24 \alpha}, \rho_{24 \alpha}$ & $H, c^{\mathrm{a}}$ & $h_{2}$ & $\rho_{12}$ & $\rho_{13 \alpha}$ \\
\hline$\nabla^{2} g^{\alpha}$ & $Y_{\alpha}, y_{\alpha}$ & $T_{2 \alpha \beta \gamma}, t_{2 \alpha \beta \gamma}$ & $P_{26 \alpha \beta}, \rho_{26 \alpha \beta}$ & $P_{24 \alpha}, \rho_{24 \alpha}$ & $P_{22 \alpha \beta}, \rho_{22 \alpha \beta}$ & $A_{5 \alpha}, a_{5 \alpha}$ & $a_{4 \alpha}$ & $\rho_{7 \alpha}$ & $\rho_{21 \alpha \beta}$ \\
\hline$R$ & $Q, \chi_{4}$ & $Y_{5 \alpha \beta}, y_{5 \alpha \beta}$ & $Q_{1 \alpha}, \chi_{\alpha}$ & $H, c^{\mathrm{a}}$ & $A_{5 \alpha}, a_{5 \alpha}$ & $A, a$ & $n$ & $h_{1}$ & $a_{7 \alpha}$ \\
\hline$\nabla^{2} \sigma$ & $\chi_{3}$ & $a_{3 \alpha \beta}$ & $\chi_{1 \alpha}$ & $h_{2}$ & $a_{4 \alpha}$ & $n$ & $\mathrm{NA}$ & $\mathrm{NA}$ & $\mathrm{NA}$ \\
\hline$\partial_{i} \sigma \partial^{i} N$ & $\rho_{11}$ & $\rho_{1 \alpha \beta}$ & $\rho_{10 \alpha}$ & $\rho_{12}$ & $\rho_{7 \alpha}$ & $h_{1}$ & $\mathrm{NA}$ & $\mathrm{NA}$ & $\mathrm{NA}$ \\
\hline$\partial_{i} \sigma \partial^{i} g^{\alpha}$ & $\rho_{8 \alpha}$ & $t_{\alpha \beta \gamma}$ & $x_{1 \alpha \beta}$ & $\rho_{13 \alpha}$ & $\rho_{21 \alpha \beta}$ & $a_{7 \alpha}$ & $\mathrm{NA}$ & $\mathrm{NA}$ & NA \\
\hline
\end{tabular}

${ }^{\mathrm{a}} R \nabla^{2} N$ can be written as $\partial_{i} R \partial^{i} N$ by integration by parts, and it is for the operator $\partial_{i} R \partial^{i} N$ that we use the coefficient $c$.

Table 2. Summary of four spatial derivative terms that can enter the counterterm functional $W_{\text {c.t. }}$. or the anomaly on the right hand side of eq. (2.21). The terms in $W_{\text {c.t. }}$ are the products of the first six entries of the first column and the first six of the first row, and their coefficients are the first of the entries listed in the table (uppercase letters). Those in the anomaly extend over the whole table; in the first $6 \times 6$ block they correspond to the second entry (lowercase characters) and for those a factor of $\sigma$ is implicit. The red $N A$ labels denote terms that are second order in infinitesimal parameter $\sigma$, hence dropped. Latin indices are contracted with the inverse metric $h^{i j}$ when repeated, eg, $\partial_{i} N \partial^{i} N=h^{i j} \partial_{i} N \partial_{j} N$.

\begin{tabular}{|c|c|c|c|}
\hline Operators & $K$ & $g^{\alpha}$ & $\left(K_{i j}-\frac{1}{2} K h_{i j}\right)$ \\
\hline Length Dimension & 0 & 0 & 0 \\
\hline Time Dimension & 1 & 0 & 1 \\
\hline
\end{tabular}

Table 3. Basic building blocks for operators in the $\partial_{t}^{2}$ sector and their dimensions.

\begin{tabular}{|c|c|c|c|c|}
\hline$\partial_{t}^{2}$ Sector & $K$ & $\partial_{t} g^{\alpha}$ & $\partial_{t} \sigma$ & $K_{i j}-\frac{1}{2} K h_{i j}$ \\
\hline$K$ & $D, d$ & $W_{\alpha}, w_{\alpha}$ & $f$ & NA \\
\hline$\partial_{t} g^{\alpha}$ & $W_{\alpha}, w_{\alpha}$ & $X_{0 \alpha \beta}, \chi_{0 \alpha \beta}$ & $b_{\alpha}$ & NA \\
\hline$\partial_{t} \sigma$ & $f$ & $b_{\alpha}$ & NA & NA \\
\hline$K_{i j}-\frac{1}{2} K h_{i j}$ & NA & NA & NA & $E, e$ \\
\hline
\end{tabular}

Table 4. Summary of two time derivative terms that can enter the counterterm functional $W_{\text {c.t. }}$. or the anomaly on the right hand side of eq. (2.21). The terms in $W_{\text {c.t. }}$ are the products of the first, second, fourth entries of the first column and the first, second, fourth entry of the first row , and their coefficients are the first of the entries listed in the table (uppercase letters). Those in the anomaly extend over the whole table; in the first $2 \times 2$ block they correspond to the second entry (lowercase characters) and for those a factor of $\sigma$ is implicit. The red NA labels denote terms that are either second order in infinitesimal parameter $\sigma$ or terms that are not rotationally invariant. 


\begin{tabular}{|c|c|c|c|c|c|c|c|c|c|}
\hline$\partial_{t} \nabla^{2}$ Sector & $\partial^{i} N \partial^{j} N$ & $\partial^{i} g^{\alpha} \partial^{j} g^{\beta}$ & $\partial^{i} N \partial^{j} g^{\alpha}$ & $\nabla^{i} \nabla^{j} N$ & $\nabla^{i} \nabla^{j} g^{\alpha}$ & $R$ & $\nabla^{i} \nabla^{j} \sigma$ & $\partial^{i} \sigma \partial^{j} N$ & $\partial^{i} \sigma \partial^{j} g^{\alpha}$ \\
\hline $\mathrm{K}$ & $P, \rho_{4}$ & $X_{5 \alpha \beta}, x_{5 \alpha \beta}$ & $P_{\alpha}, \rho_{\alpha}$ & $L, j^{\mathrm{a}}$ & $P_{3 \alpha}, b_{8 \alpha}$ & $B, b$ & $m$ & $l_{1}$ & $b_{7 \alpha}$ \\
\hline$\partial_{t} g^{\alpha}$ & $X_{\alpha}, \rho_{6 \alpha}$ & $X_{3 \alpha \beta \gamma}, x_{3 \alpha \beta \gamma}$ & $P_{4 \alpha \beta}, p_{4 \alpha \beta}$ & $B_{6 \alpha}, b_{6 \alpha}$ & $X_{4 \alpha \beta}, x_{4 \alpha \beta}$ & $B_{5 \alpha}, b_{5 \alpha}$ & $B_{9 \alpha}, b_{9 \alpha}$ & $\rho_{5 \alpha}$ & $x_{6 \alpha \beta}$ \\
\hline$\partial_{t} \sigma$ & $\rho_{3}$ & $b_{3 \alpha \beta}$ & $\rho_{1 \alpha}$ & $l_{2}$ & $b_{4 \alpha}$ & $k$ & $\mathrm{NA}$ & $\mathrm{NA}$ & $\mathrm{NA}$ \\
\hline$K_{i j}-\frac{1}{2} K h_{i j}$ & $F_{1}, f_{1}$ & $F_{2 \alpha \beta}, f_{2 \alpha \beta}$ & $F_{3 \alpha}, f_{3 \alpha}$ & $F_{4}, f_{4}$ & $F_{5 \alpha}, f_{5 \alpha}$ & $\mathrm{NA}$ & $f_{6}$ & $f_{7}$ & $f_{8 \alpha}$ \\
\hline
\end{tabular}

${ }^{\mathrm{a}} K \nabla^{2} N$ can be written as $\partial_{i} K \partial^{i} N$ by doing integration by parts, and it is for this operator that we use the coefficient $j$.

Table 5. Summary of one-time, two-space derivative terms that can enter the counterterm functional $W_{\text {c.t. }}$ or the anomaly on the right hand side of eq. (2.21). The terms in $W_{\text {c.t. }}$ are the products of the entries that have no explicit $\sigma$ factor, and their coefficients are the first of the entries listed in the table (uppercase letters). Those in the anomaly extend over the whole table; terms without explicit $\sigma$ have coefficients that correspond to the second entry (lowercase characters) and for those a factor of $\sigma$ must be included. Latin indices are contracted with the spatial metric as necessary to make the product of the first column and first row entries rotationally invariant; for example, $\rho_{4}$ denotes the coefficient of $K \partial_{i} N \partial^{i} N$. For last entry in the first column, indices are contracted with those in the terms in first row. The red NA labels denote terms that are second order in infinitesimal parameter $\sigma$, hence dropped. The blue NA one denotes a term that is identically 0 since $K_{i j}-\frac{1}{2} K h_{i j}$ vanishes upon contraction via $h^{i j}$.

with itself. Thus in total there are $3+2+1=6$ terms listed in table 4 that also gives the corresponding coefficients.

The sector with mixed derivatives has terms with one time and two spatial derivatives. For this $\partial_{t} \nabla^{2}$ sector we can form terms by combining one of (3.4) or (3.5) with one of (3.1), (3.2) or (3.3), excluding terms quadratic in $\sigma$. This gives $3 * 9-3=24$ terms, as displayed with their coefficients in table 5. Finally, we have terms that are not constructed as products of rotationally invariant quantities. Coefficient of those terms are listed in the last row of table 5 .

\subsection{Using counter-terms}

One can similarly list all possible terms in $W_{\text {c.t. }}$ The requirements imposed by dimensional analysis and rotational invariance are as before, the only difference being that these terms are built from the metric and the couplings but not the parameter of the Weyl transformation $\sigma$. Therefore the list of possible counterterms is obtained from the one for anomalies by replacing $\sigma \rightarrow 1$. Tables. 2, 4 and 5 give, as uppercase letters, our notation for the coefficients of these operators in $W_{\text {c.t. }}$.

The counterterms in $W_{\text {c.t. }}$ are not completely fixed by requiring finiteness of the generating functional. The ambiguity consists of the freedom to include arbitrary finite contributions to each term. This freedom to add finite counter-terms does not affect the consistency conditions but does change the value of the individual terms related by them. We can use this freedom to set some anomalies to zero, simplifying the analysis of the consequences of the Weyl consistency conditions. In particular, in searching for an $a$-theorem we can use this freedom to simplify the consistency conditions. It may be possible to show then that there exist some class of subtraction schemes for which there exists a possible candidate 


\begin{tabular}{|c|c|}
\hline Sector & Trivial Anomalies \\
\hline$\partial_{t}^{2}$ & $f, b_{\alpha}$ \\
\hline$\nabla^{2} \partial_{t}$ & $\left(\rho_{3}, l_{1}\right), x_{6 \alpha \beta}, \rho_{5 \alpha}, b_{3 \alpha \beta}, b_{4 \alpha}, b_{9 \alpha},\left(k, m, l_{2}\right),\left(b_{7 \alpha}, \rho_{1 \alpha}\right), f_{6}, f_{7}, f_{8 \alpha}$ \\
\hline$\nabla^{4}$ & $\chi_{3}, \rho_{11},\left(\rho_{10 \alpha}, \rho_{13 \alpha}, \rho_{8 \alpha}\right), a_{3 \alpha \beta}, \rho_{1 \alpha \beta}, t_{\alpha \beta \gamma}, \chi_{1 \alpha}, x_{1 \alpha \beta}, h_{2}, \rho_{12}, a_{4 \alpha}, \rho_{7 \alpha}, \rho_{21 \alpha \beta}, n, h_{1}, a_{7 \alpha}$ \\
\hline
\end{tabular}

Table 6. Trivial anomalies for each sector. Finite ambiguities in counter-terms give sufficient freedom to set all these anomalies arbitrarily; setting them to zero is often convenient. For anomalies grouped within parenthesis, all but one of them can be set arbitrarily.

for an $a$-theorem, but a general, counter-term and scheme independent statement may not be possible.

To illustrate this, consider the variation of the $K^{2}$ and $K \partial_{t} g^{\alpha}$ terms in $W_{\text {c.t. }}$ :

$$
\begin{aligned}
& \Delta_{\sigma} \int d t d^{2} x N \sqrt{h}\left(D K^{2}\right)=\int d t d^{2} x N \sqrt{h}\left(-4 \frac{1}{N} \partial_{t} \sigma D K-\sigma \beta^{\alpha} \partial_{\alpha} D K^{2}\right), \\
& \Delta_{\sigma} \int d t d^{2} x N \sqrt{h}\left(W_{\alpha} K \partial_{t} g^{\alpha}\right)=\int d t d^{2} x N \sqrt{h}\left(-\sigma\left[\beta^{\alpha} \partial_{\alpha} W_{\gamma}+W_{\alpha} \partial_{\gamma} \beta^{\alpha}\right] K \partial_{t} g^{\gamma}\right. \\
&\left.-\frac{1}{N} \partial_{t} \sigma \beta^{\alpha} W_{\alpha} K-2 \frac{1}{N} \partial_{t} \sigma W_{\alpha} \partial_{t} g^{\alpha}\right)
\end{aligned}
$$

Inspecting tables 2, 4 and 5 we see that the $f$ anomaly gets contributions only from these variations, so that the change in $f$ induced by finite changes in the counterterms is given by

$$
\delta f=-4 D-\beta^{\alpha} W_{\alpha} .
$$

With a slight abuse of notation we have denoted here the arbitrary, finite, additive change to the coefficients of counterterms by the same symbol we have used for the counterterm coefficients themselves. From eq. (3.7) we see that one can always choose $D$ so as to set $f$ arbitrarily, and it is often convenient to set $f=0$. For a second example consider the $R^{2}$ anomaly, $a$. A similar computation gives

$$
\delta a=-\beta^{\alpha} \partial_{\alpha} A
$$

In this case we may solve this equation so as to set $a=0$ only if $a=0$ at fixed points, where $\beta^{\alpha}=0$. As we will see below, the Weyl consistency conditions constrain some anomalies to vanish at fixed points.

We give in appendix $\mathrm{B}$ the complete set of ambiguities for models with $z=2$ in $d=2$ spatial dimensions. Terms in the effective actions whose coefficients can be varied at will are not properly anomalies, since the coefficients can be set to zero. With a slight abuse of language they are commonly referred to as trivial anomalies and we adopt this terminology here. Table 6 summarizes the trivial anomalies found in each sector.

\subsection{Consistency conditions and vanishing anomalies}

In computing the consistency condition (2.23) one finds a functional that is a combination of linearly independent "operators" (combinations of $\sigma, \gamma_{\mu \nu}$ and $g^{\alpha}$ ), each with a coefficient 


\begin{tabular}{|c|c|c|}
\hline Sector & Vanishing Anomalies & $\begin{array}{c}\text { Conditionally } \\
\text { Vanishing Anomalies }\end{array}$ \\
\hline$\partial_{t}^{2}$ & $d$ & $w_{\alpha}$ \\
\hline$\nabla^{2} \partial_{t}$ & $f_{4}, f_{1}, \rho_{4}, b_{7 \alpha}$ & $b_{6 \alpha}+\rho_{6 \alpha}, b_{5 \alpha}-b_{6 \alpha}, b_{7 \alpha}-\rho_{1 \alpha}$ \\
& $b, j, 2 \rho_{3}-l_{1}+2 l_{2}, k+m-l_{2}$ & $x_{5 \alpha \beta}, f_{3 \alpha}, b_{8 \alpha}$ \\
\hline$\nabla^{4}$ & $\chi_{4}-p_{4}, 2 p_{3}+p_{4}, c-\chi_{4}, h_{1}+2 h_{2}+2 \chi_{3}-c-\rho_{12}$ & $x_{\alpha \beta}+x_{2 \alpha \beta}, \rho_{13 \alpha}$ \\
& $2 a+c, p_{4}+2 \rho_{23}, 2 \rho_{23}+c$ & $y_{5 \alpha \beta}-x_{2 \alpha \beta}, y_{\alpha}+\rho_{24 \alpha}, a_{5 \alpha}-\rho_{24 \alpha}, \rho_{25 \alpha}+\rho_{9 \alpha}$ \\
\hline
\end{tabular}

Table 7. Vanishing anomalies for each sector. The Weyl consistency conditions imply these anomalies, or combination of anomalies, vanish at fixed points (where $\beta^{\alpha}=0$ ). An anomaly is conditionally vanishing if it is vanishing only for a particular choice of counterterms.

that is a linear combination of the coefficients in tables 2, 4 and 5 and their derivatives. Thus the consistency conditions can be expressed as a set of equations among these coefficients and their derivatives. The full set of consistency conditions for $d=2, z=2$ are listed in appendix A. On the left of each condition we have listed the operator the condition arises from. We have verified that these conditions reduce to the ones computed in ref. [23] at fixed points. In the $\partial_{t} \nabla^{2}$ sector the consistency conditions, eqs. (A.1), are given for arbitrary $z$, while for the $\partial_{t}^{2}$ and $\nabla^{4}$ sectors, eqs. (A.2) and (A.3), respectively, the value $z=2$ has been used.

At fixed points the consistency conditions imply some anomalies vanish. These are known as vanishing anomalies. For example, setting $\beta^{\alpha}=0$ in eq. (A.2a) gives $d=0$. Table 7 summarizes the vanishing anomalies found in each sector. The table also shows conditionally vanishing anomalies. These are vanishing anomalies but only for a specific choice of counterterms. For example, setting $\beta^{\alpha}=0$ in eq. (A.2b) gives $-2 w_{\alpha}+b_{\gamma} \partial_{\alpha} \beta^{\gamma}=0$, and eq. (B.1c) shows that we can choose the counterterm $W_{\alpha}$ to set $b_{\alpha}=0$.

As explained above, some vanishing anomalies can be set to zero. For example, from table 7 we see that $d$ is a vanishing anomaly, and then eq. (B.1e) informs us that one may choose $D$ to enforce $d=0$. We note, however, that by eqs. (B.1a) and (B.1e) one may either choose $f$ or $d$ to vanish, but not both.

\subsection{Applications}

While there are many avenues for analysis in light of the relations imposed by Weyl consistency conditions on the anomalies, we concentrate on finding candidates for a C-theorem. We search for a combination of anomalies, $C$, a local function in the space of dimensionless coupling constants that flows monotonically, $\mu d C / d \mu \geq 0$. We try to establish this by judiciously setting some anomalies to zero by the freedom explained above and looking for a relation of the form

$$
\beta^{\alpha} \partial_{\alpha} C=-\mathcal{H}_{\alpha \gamma} \beta^{\alpha} \beta^{\gamma} .
$$

Our first three candidates arise from the $\nabla^{4}$ sector. Consider eq. (A.3l), here reproduced:

$$
-a_{5 \alpha} \beta^{\alpha}+4 a+2 c+\beta^{\alpha} \partial_{\alpha} n=0
$$


The combination $2 a+c$ is a vanishing anomaly. One may then use (B.3aj) and (B.3aa) to set $2 a+c=0$. Equation (B.3ag) shows $a_{4 \alpha}$ is a trivial anomaly and one may set $a_{4 \alpha}=0$. Combining with eq. (A.3c) we have

$$
\beta^{\alpha} \partial_{\alpha} n=\rho_{22 \alpha \gamma} \beta^{\alpha} \beta^{\gamma}+\rho_{24 \alpha} \beta^{\alpha}
$$

Similarly, eq. (A.3i) shows $2 \rho_{23}+c$ is a vanishing anomaly and using (B.3y) we may set $2 \rho_{23}+c=0$. We then have from eq. (A.3i) again that

$$
\beta^{\gamma} \partial_{\gamma} h_{2}=\beta^{\gamma} \rho_{24 \gamma}
$$

The difference of these equations then gives us our first candidate for a C-theorem, with $C=n-h_{2}$ :

$$
\beta^{\alpha} \partial_{\alpha}\left(n-h_{2}\right)=\rho_{22 \alpha \gamma} \beta^{\alpha} \beta^{\gamma} .
$$

A second candidate can be found as follows. Eq. (A.3s) shows $\chi_{4}-p_{4}$ is a vanishing anomaly. Then $Q-P_{4}$ can be chosen so that $\chi_{4}-p_{4}=0$; see eqs. (B.3f) and (B.3d). Using eq. (A.3k) with $\rho_{7 \alpha}=0$ as it is a trivial anomaly, we obtain

$$
-\beta^{\alpha} \partial_{\alpha} \chi_{3}=\frac{1}{4} \rho_{26 \alpha \gamma} \beta^{\alpha} \beta^{\gamma}+\rho_{24 \alpha} \beta^{\alpha}
$$

It follows that

$$
\beta^{\alpha} \partial_{\alpha}\left(n+\chi_{3}\right)=\left(\rho_{22 \alpha \gamma}-\frac{1}{4} \rho_{26 \alpha \gamma}\right) \beta^{\alpha} \beta^{\gamma}
$$

Combining eqs. (A.3n), (A.3j) and (A.3r) while setting $\chi_{1 \alpha}=0, p_{4}+2 \rho_{23}=0$ and $c-\chi_{4}=0$ gives what appears to be yet another candidtae in the $\nabla^{4}$ sector:

$$
\beta^{\alpha} \partial_{\alpha}\left(c+\rho_{12}-h_{1}\right)=-\frac{1}{2} \rho_{26 \alpha \gamma} \beta^{\alpha} \beta^{\gamma}
$$

However, setting the trivial anomalies $\rho_{1 \alpha}$ and $\chi_{1 \alpha}$ to zero, eq. (A.3o) gives

$$
h_{2}+\chi_{3}=\frac{1}{2}\left(c+\rho_{12}-h_{1}\right)
$$

which shows that the candidates given by eq (3.9), (3.10), (3.11) are not linearly independent in the scheme with $2 a+c=2 \rho_{23}+c=\chi_{4}-c=\chi_{4}-p_{4}=p_{4}+\rho_{23}=0$ and $a_{4 \alpha}=\rho_{1 \alpha}=\rho_{7 \alpha}=\chi_{1 \alpha}=0$.

We find one candidate for a C-theorem in the $\partial_{t}^{2}$ sector. Equation (A.2a) shows $d$ is a vanishing anomaly and use eqs. (B.1e) and (B.1c) to set $d=b_{\alpha}=0$. Combining (A.2a) and (A.2b) gives

$$
\beta^{\alpha} \partial_{\alpha} f=-\chi_{0 \alpha \gamma} \beta^{\alpha} \beta^{\gamma} .
$$

In the $\partial_{t} \nabla^{2}$-sector we find the following candidates for a $C$-theorem:

$$
\begin{aligned}
\beta^{\alpha} \partial_{\alpha} m & =-\frac{1}{2} x_{4 \alpha \gamma} \beta^{\alpha} \beta^{\gamma} \\
\beta^{\alpha} \partial_{\alpha} l_{1} & =-\frac{1}{2} p_{4 \gamma \alpha} \beta^{\gamma} \beta^{\alpha} \\
\beta^{\alpha} \partial_{\alpha}\left(\rho_{3}+l_{2}\right) & =-\frac{1}{2 z} p_{4 \alpha \gamma} \beta^{\gamma} \beta^{\alpha} \\
\beta^{\alpha} \partial_{\alpha}\left(f_{6}+\frac{z}{2} f_{7}-\beta^{\gamma} f_{5 \gamma}\right) & =\beta^{\alpha} \beta^{\gamma}\left(f_{2 \alpha \gamma}-\partial_{\alpha} f_{5 \gamma}\right)
\end{aligned}
$$


We have kept the explicit dependence on $z$ in these equations. As we will see below the $\partial_{t} \nabla^{2}$-sector is special in that the Weyl anomalies and the relations from consistency conditions hold for arbitrary $z$. Hence, the $C$-candidates in this sector are particularly interesting since they are candidates for any $z$. To derive (3.13) we have used that $j$ and $b$ are vanishing anomalies, as evident from eqs. (A.1d) and (A.1f), and used $B$ and $L$ to set $b=j=0$ in eq. (A.1f) and $P_{3 \alpha}$ to set $b_{4 \alpha}=0$ in eq. (A.1b). For (3.14) we used $j=0$ in eq. (A.1a) and (A.1n), deduce that $\rho_{4}$ is a vanishing anomaly and use $P$ to set $\rho_{4}=0$ in eq. (A.1n) and $P_{\alpha}$ to set $\rho_{1 \alpha}=0$ in eq. (A.1a). For (3.15), we set $j=\rho_{4}=0$ as before and in addition we set $\rho_{5 \alpha}=0$ using $X_{\alpha}$ in (A.1e), and use eqs. (A.1d), (A.1e) and (A.1m). In the scheme, $j=\rho_{1 \alpha}=0, \mathrm{Eq}$ (A.1o) implies that the candidates given by (3.15) and (3.14) are linearly dependent. Last but not the least, (3.16) is derived from eqs. (A.1p)-(A.1r) by using $F_{3 \alpha}$ to set $f_{8 \alpha}=0$ and setting to zero the vanishing anomalies $f_{1}$ and $f_{4}$ using $F_{1}$ and $F_{4}$.

Two comments are in order. First, we have not established any $C$-theorem. To do so would require showing that the two index symmetric tensor appearing on at least one of the right hand side of eqs. (3.9)-(3.12) is positive definite, so that it acts as a metric in the space of flows. In addition, the interpretation of $C$ as counting degrees of freedom is better supported if it is a monotonic function of the number of degrees of freedom at a gaussian fixed point. And second, we do not expect a positive definite metric can be found in generality, since cyclic flows are known to appear in NR quantum systems. Cyclic flows appear in relativistic systems too, but they differ from NR ones in that there is scaling symmetry all along the cyclic flows and, in fact, the $C$ quantity is constant along the cyclic flow [45]. Investigating the conditions under which a theory gives positive definite metric(s) in the space of flows is beyond the scope of this work; we hope to return to this problem in the future.

\section{Generalisation to arbitrary $z$ value}

In this section, we will explore the possibility to generalize the work for arbitrary $z$ value. It is clear that the formalism fails for non-integer values of $z$ since in that case, we can not make up for dimensions with regular analytic functions of curvature and coupling constants. This is because the quantities constructed out of geometry and coupling constants always have integer length and time dimension. Furthermore, in a Lagrangian formulation a noninteger $z$ requires non-analyticity of Lagrangian. So we begin by recalling under what conditions a Lagrangian with local interactions allows for integer $z$ values.

Consider first the case of $d=2$ at arbitrary $z$ value. In constructing $\Delta W_{\text {c.t. }}$, rotational invariance implies even number of spatial derivatives, say $2 n$. Along with $m$ time derivatives, we must have

$$
m z+2 n=z+2 .
$$

We look for solutions with integer values for $m$ and $n$. For $m=1$ we must have $n=1$ and this satisfies the equation for any $z$. Else, for $m \neq 1$ we have

$$
z=\frac{2(1-n)}{(m-1)}
$$


For $z>0$ we must have either $m=0$ with $n>1$ or $n=0$ with $m>1$. For $m=0$ solutions exist only if $z=2 k$ is even, with $2 n=2(1+k)$ spatial derivatives. On the other hand, with $n=0$, we have solutions for $z=2 / k$, with $m=k+2$ time derivatives. To summarize, for $z>0$ we can classify the counterterms by sector as follows:

- There is a pure $\nabla^{2}$ sector for $z=2 k, k \in \mathbf{Z}$. It has precisely $2(k+1)$ spatial derivatives. We have discussed in detail the case $k=1$. Higher values of $k$ can be similarly analyzed, but it it involves an ever increasing number of terms as $z$ increases.

- There is a pure $\partial_{t}$ sector for $z=2 / k, k \in \mathbf{Z}$, with $k+1$ time derivatives. We have analyzed the $k=1$ case. Higher values of $k$ can be similarly analyzed, but it involves an ever increasing number of terms as $z$ decreases.

- There is a $\partial_{t} \nabla^{2}$ sector for arbitrary $z$. It has 1-time and 2-spatal derivatives regardless of $z$. Therefore, the classification of anomalies and counterterms is exactly as in the $z=2$ case, and the consistency conditions and derived $C$-candidates are modified by factors of $z / 2$ relative the $z=2$ case.

\section{A candidate for a $C$-theorem in $d+1 \mathrm{D}$}

In relativistic $2 n$-dimensional QFT the quantity that is believed to satisfy a $C$-theorem is associated with the Euler anomaly, that is, it is the coefficient of the Euler density $E_{2 n}$ in the conformal anomaly [33]. ${ }^{2}$ It would seem natural to seek for analogous candidates in nonrelativistic theories. The obvious analog involves the Euler density for the spatial sections $t=$ constant; by dimensional analysis and scaling it should be constructed out of $z+d=2 n$ spatial derivatives acting on the metric $h_{i j}$. However, for a $d$-dimensional metric the Euler density $E_{2 n}$ with $2 n-d=z>0$ vanishes. Hence, we are led to consider an anomaly of the form $X E_{d}$, that is the Euler density computed on the spatial sections $t=$ constant times some quantity $X$ with the correct dimensions, $[X]=z$. This construction is only valid for even spatial dimension, $d=2 n$. The most natural candidate for $X$ is $K$ : it is the only choice if $z$ is odd. If $z$ is even it can be constructed out of spatial derivatives. For example, if $z=d k=2 n k$ for some integers $k$ and $n$, one may take $X=\left(E_{d}\right)^{k}$.

The variation of the Euler density yields the Lovelock tensor [52], $H_{i j}$, a symmetric 2-index tensor that satisfies

$$
\nabla_{i} H^{i j}=0
$$

In looking for a candidate $C$-theorem we consider a set of operators that close under Weyl-consistency conditions, starting from $X E_{d}$. Since $\delta_{\sigma}\left(\sqrt{h} E_{d}\right)=\sqrt{h} H^{i j} \nabla_{i} \partial_{j} \sigma$, and $\left[X H^{i j}\right]=z+d-2$, we are led to include terms with the Lovelock tensor and two spatial derivatives. In order to compute the consequences of the Weyl consistency conditions we assume

$$
\delta X=z \sigma X+\cdots
$$

\footnotetext{
${ }^{2}$ There is no known local $C$-function candidate for odd-dimensional relativistic field theory. Jafferis has proposed a non-local $F$-function for 3D relativistic theories that shares the monotonicity properties of a $C$-function [51].
} 
where the ellipses denote terms that depend on derivatives of $\sigma$ and are therefore independent of $X$. Consider therefore a subset of terms in the anomaly that appear in the consistency conditions that lead to a potential $C$-theorem:

$$
\begin{aligned}
\Delta_{\sigma} W=\int d^{d} x d t N \sqrt{h}[\sigma\{ & a X E_{d}+b X H^{i j} R_{i j}+\chi_{4} X H^{i j} \frac{\partial_{i} N}{N} \frac{\partial_{j} N}{N}+\chi_{\alpha} X H^{i j} \frac{\partial_{i} N}{N} \partial_{j} g^{\alpha} \\
& \left.+y_{5 \alpha \beta} X H^{i j} \partial_{i} g^{\alpha} \partial_{j} g^{\beta}+c H^{i j} \partial_{i} X \frac{\partial_{i} N}{N}+a_{5 \alpha} H^{i j} \partial_{i} X \partial_{j} g^{\alpha}\right\} \\
& \left.+\partial_{i} \sigma\left\{n \partial_{j} X H^{i j}+h_{1} \frac{\partial_{j} N}{N} H^{i j} X+a_{7 \alpha} \partial_{j} g^{\alpha} H^{i j} X\right\}\right]
\end{aligned}
$$

Correspondingly there are metric and coupling-constant dependent counter-terms with coefficients denoted by uppercase symbols:

$$
\begin{aligned}
W_{\text {c.t. }}=\int d^{d} x d t N \sqrt{h}[ & A X E_{d}+B X H^{i j} R_{i j}+X_{4} X H^{i j} \frac{\partial_{i} N}{N} \frac{\partial_{j} N}{N}+X_{\alpha} X H^{i j} \frac{\partial_{i} N}{N} \partial_{j} g^{\alpha} \\
& \left.+Y_{5 \alpha \beta} X H^{i j} \partial_{i} g^{\alpha} \partial_{j} g^{\beta}+C H^{i j} \partial_{i} X \frac{\partial_{i} N}{N}+A_{5 \alpha} H^{i j} \partial_{i} X \partial_{j} g^{\alpha}\right]
\end{aligned}
$$

Freedom to choose finite parts of counter-terms leads to ambiguities in the anomaly coefficients as follows:

$$
\begin{aligned}
\delta a & =-\beta^{\alpha} \partial_{\alpha} A \\
\delta \chi_{4} & =-\beta^{\alpha} \partial_{\alpha} X_{4} \\
\delta \chi_{\alpha} & =-\beta^{\gamma} \partial_{\gamma} X_{\alpha}-X_{\gamma} \partial_{\alpha} \beta^{\gamma} \\
\delta y_{5 \alpha \beta} & =-\beta^{\gamma} \partial_{\gamma} Y_{5 \alpha \beta}-Y_{5 \gamma \beta} \partial_{\alpha} \beta^{\gamma}-Y_{5 \alpha \gamma} \partial_{\beta} \beta^{\gamma} \\
\delta c & =-\beta^{\alpha} \partial_{\alpha} C \\
\delta b & =-\beta^{\alpha} \partial_{\alpha} B \\
\delta a_{5 \alpha} & =-\beta^{\gamma} \partial_{\gamma} A_{5 \alpha}-A_{5 \gamma} \partial_{\alpha} \beta^{\gamma} \\
\delta n & =-A-(d-2) B-C z-\beta^{\alpha} A_{5 \alpha} \\
\delta h_{1} & =-2 z X_{4}-\beta^{\alpha} X_{\alpha}+C z-A-(d-2) B \\
\delta a_{7 \alpha} & =-\partial_{\alpha}(A+(d-2) B)-z X_{\alpha}-2 \beta^{\gamma} Y_{5 \gamma \alpha}+z A_{5 \alpha}
\end{aligned}
$$

In addition to the Euler density, $E_{d}$, there are several independent scalars one can construct out of $d$ derivatives of the metric in $d$ dimensions (except for $d=2$, for which the only 2-derivative invariant is the Ricci scalar and hence $\left.E_{d} \propto R\right) . E_{d}$ is special in that it is the only quantity that gives just the Lovelock tensor under an infinitesimal Weyl trasformation, $\delta_{\sigma}\left(\sqrt{h} E_{d}\right)=\sqrt{h} H^{i j} \nabla_{i} \partial_{j} \sigma$. In general some other $d$-derivative invariant ${ }^{3} \mathcal{E}$ constructed out of $d / 2$ powers of the Riemann tensor will instead give $\delta_{\sigma}(\sqrt{h} \mathcal{E})=\sqrt{h} \mathcal{H}^{i j} \nabla_{i} \partial_{j} \sigma$ where $\mathcal{H}^{i j} \neq 0$ is not divergence-less, $\nabla_{i} \mathcal{H}^{i j} \neq 0$. We have given an example of such a term above, $H^{i j} R_{i j}$, both in the anomaly and among the counter-terms. Given a basis of $d$ derivative operators $\mathcal{E}$ and $d-2$ derivative 2-index symmetric tensors $\mathcal{H}^{i j}$ one can derive

\footnotetext{
${ }^{3}$ Weyl variations of $d$-derivative scalars constructed from less than $d / 2$ powers of the Riemann tensor do not contribute to the consistency condition we are considering.
} 
Weyl consistency conditions by demanding that the coefficients of each linearly independent operator in $\left[\Delta_{\sigma}, \Delta_{\sigma^{\prime}}\right] W$ vanish. Suppose $\Delta_{\sigma} W \supset \int \sigma\left[a E_{d}+b \mathcal{E}\right]$ : a change of basis by $\mathcal{E} \rightarrow \mathcal{E}+\xi E_{d}$ results in shifting $a \rightarrow a+\xi b$ in the consistency conditions that arise from terms involving $H^{i j}$. Similarly, a change of basis of $d-2$ derivative 2-index symmetric tensors $\mathcal{H}^{i j} \rightarrow \mathcal{H}^{i j}+\xi H^{i j}$ shifts by a common amount all the consistency conditions that arise from terms involving $H^{i j}$. So while we have not retained all the anomalies that can contribute to the consistency conditions that lead to a potential $C$-theorem, they give a common contribution to all those consistency conditions and therefore effectively shift the contribution of $a$ to the potential $C$-theorem - and the shift is immaterial since it is basis dependent. Consider, for example, the coefficient $b$ of the anomaly term $H^{i j} R_{i j}$ which we have retained precisely to demonstrate these points. Since $\delta_{\sigma} R_{i j}=(d-2) \nabla_{i} \partial_{j} \sigma+h_{i j} \nabla^{2} \sigma$ it is natural to define $\mathcal{H}_{i j}$ by $\delta_{\sigma}\left(\sqrt{h} H^{i j} R_{i j}\right)=\sqrt{h}\left[(d-2) H^{i j}+\mathcal{H}^{i j}\right] \nabla_{i} \partial_{j} \sigma$. With this definition of a basis of operators the consistency conditions in eqs. (5.5) below all contain the combination $a+(d-2) b$; had we defined instead a basis with the operator $H^{i j} R_{i j}-(d-2) E_{d}$ or defined the basis of 2-index tensors through $\delta_{\sigma}\left(\sqrt{h} H^{i j} R_{i j}\right)=\sqrt{h} \mathcal{H}^{i j} \nabla_{i} \partial_{j} \sigma$, the anomaly $b$ would not have appeared in eqs. (5.5) at all. Similarly the ambiguity due to finite counter-terms in anomalies associated with the Lovelock tensor all enter in the combination $A+(d-2) B$.

Imposing $\left[\Delta_{\sigma^{\prime}}, \Delta_{\sigma}\right] W=0$ we find three conditions,

$$
\begin{aligned}
\left(\sigma \partial_{j} \sigma^{\prime}-\sigma^{\prime} \partial_{j} \sigma\right) H^{i j} \partial_{i} X: & \beta^{\alpha} \partial_{\alpha} n=z c+a_{5 \alpha} \beta^{\alpha}+a+(d-2) b \\
\left(\sigma \partial_{j} \sigma^{\prime}-\sigma^{\prime} \partial_{j} \sigma\right) H^{i j} \partial_{i} N X: & \beta^{\alpha} \partial_{\alpha} h_{1}=a+(d-2) b+2 z \chi_{4}+\beta^{\alpha} \chi_{\alpha}-c z \\
\left(\sigma \partial_{j} \sigma^{\prime}-\sigma^{\prime} \partial_{j} \sigma\right) H^{i j} \partial_{i} g^{\alpha} X: & \partial_{\alpha}(a+(d-2) b)-\beta^{\gamma} \partial_{\gamma} a_{7 \alpha}-a_{7 \gamma} \partial_{\alpha} \beta^{\gamma}=z a_{5 \alpha}-z \chi_{\alpha}-2 y_{5 \alpha \gamma} \beta^{\gamma}
\end{aligned}
$$

Here we have listed on the left the independent operators in $\left[\Delta_{\sigma^{\prime}}, \Delta_{\sigma}\right] W$ whose coefficients must vanish yielding the condition correspondingly listed on the right. We have checked that the conditions in eqs. (5.5) are invariant under the ambiguities listed in eqs. (5.4). The freedom represented by these ambiguities allows us to set $a+(d-2) b+z c=0$ in eq. (5.5a). To see this note that $a+(d-2) b+z c$ is a vanishing anomaly per eq. (5.5a), and eqs. (5.4a), (5.4e) and (5.4f) give $\delta(a+(d-2) b+z c)=-\beta^{\alpha} \partial_{\alpha}(A+(d-2) B+z C)$ which can be integrated. A similar argument using eq. (5.5b) shows that $a+(d-2) b+$ $2 z \chi_{4}-c z$ is a vanishing anomaly. Using this freedom we have a simpler version of the consistency conditions:

$$
\begin{aligned}
\left(\sigma \partial_{j} \sigma^{\prime}-\sigma^{\prime} \partial_{j} \sigma\right) H^{i j} \partial_{i} X: & \beta^{\alpha} \partial_{\alpha} n=a_{5 \alpha} \beta^{\alpha} \\
\left(\sigma \partial_{j} \sigma^{\prime}-\sigma^{\prime} \partial_{j} \sigma\right) H^{i j} \partial_{i} N X: & \beta^{\alpha} \partial_{\alpha} h_{1}=\beta^{\alpha} \chi_{\alpha} \\
\left(\sigma \partial_{j} \sigma^{\prime}-\sigma^{\prime} \partial_{j} \sigma\right) H^{i j} \partial_{i} g^{\alpha} X: & \partial_{\alpha}(a+(d-2) b)-\beta^{\gamma} \partial_{\gamma} a_{7 \alpha}-a_{7 \gamma} \partial_{\alpha} \beta^{\gamma}=z a_{5 \alpha}-z \chi_{\alpha}-2 y_{5 \alpha \gamma} \beta^{\gamma}
\end{aligned}
$$

Combining these we arrive at the candidate for a $C$-theorem:

$$
\beta^{\alpha} \partial_{\alpha}\left[a+(d-2) b+z h_{1}-z n-\beta^{\gamma} a_{7 \gamma}\right]=-2 y_{5 \alpha \gamma} \beta^{\gamma} \beta^{\alpha}
$$

Establishing a $C$-theorem requires in addition demonstrating positivity of the "metric" $-2 y_{5 \alpha \gamma}$ in eq. (5.6). While we have not attempted this, it may be possible to demonstrate 
this in generality working on a background with positive definite Lovelock tensor and using the fact that $y_{5 \alpha \gamma}$ gives the RG response of the contact counter-term to the obviously positive definite correlator $\left\langle\mathcal{O}_{\alpha} \mathcal{O}_{\gamma}\right\rangle$. In addition, one should check that, when computed at the gaussiaan fixed point, the quantity $a+(d-2) b+z h_{1}-z n-\beta^{\gamma} a_{7 \gamma}$ is a measure of the number of degrees of freedom. We hope to come back to this questions in the future, by performing explicit calculations (at and away from fixed points) of these quantities - but such extensive computations are beyond the scope of this work.

The limit $d=2$ is special since $H^{i j}=2 h^{i j}$. In our analysis, the term $H^{i j} R_{i j}=2 R=$ $2 E_{2}$ so $a$ and $b$ appear in the combination $a+2 b$ throughout. The potential $C$ theorem reads

$$
\beta^{\alpha} \partial_{\alpha}\left[a+2 b+z h_{1}-z n-\beta^{\gamma} a_{7 \gamma}\right]=-2 y_{5 \alpha \gamma} \beta^{\gamma} \beta^{\alpha}
$$

As we have seen in section 4 , potential $C$-theorems in $d=2$ for any $z$ can be found only in the $\nabla^{2} \partial_{t}$ sector. Consulting table 5 we see the only candidate for $X$ in our present discussion is $X=K$. None of the potential $C$-theorems listed in eqs. (3.13)-(3.16) (nor linear combinations thereof) reproduce the potential $C$-theorem in eq. (5.7). The reason for this is that in section 3.4 we looked for $C$-theorems from consistency conditions that included, among others, tems with $\sigma \nabla_{i} \partial_{j} \sigma^{\prime}-\sigma^{\prime} \nabla_{i} \partial_{j} \sigma$, wheras in this section we integrated such terms by parts. The difference then corresponds to combining the consistency conditions given in the appendix with some of their derivatives.

In fact we have found a scheme for deducing aditional $C$-theorem candidates in $d=2$ by taking derivatives of some of our consistency conditions. The method is as follows. Take $X \in\left\{R, \nabla^{2} N, \partial_{i} N \partial^{i} N, K\right\}$; the first three instances apply to the case $z=2$ while the last is applicable for arbitrary $z$. Then:

- Consider the consistency condition involving $\sigma \nabla^{2} \sigma^{\prime} X$, and take a derivative to obtain an equation, say $T_{1}$.

- Take the consistency condition involving $\sigma \nabla_{i} \sigma^{\prime} \partial^{i} N X$. From this one may deduce a linear combination of anomalies is vanishing. Set that to 0 using the ambiguity afforded by counter-terms. The remaining terms in the equation (all proportional to $\left.\beta^{\alpha}\right)$ give an equation we denote by $T_{2}$.

- Take the consistency condition involving $\sigma \nabla_{i} \sigma^{\prime} \partial^{i} g^{\alpha} X$, contract it with $\beta^{\alpha}$, to get an equation, say, $T_{3}$.

- Combine $T_{1}, T_{2}, T_{3}$ in a manner such that there are no terms of the form $\beta^{\alpha} r_{\alpha \ldots}$ and $r_{\gamma \ldots \beta^{\alpha}} \partial_{\alpha} \beta^{\gamma}$.

Following this scheme we obtain four new $C$-theorem candidates. In the following the expressions for $T_{1,2,3}$ refer to the equation numbers of the consistency conditions in the appendix:

(i) $X=R . T_{1}=\mathrm{A} .31, T_{2}=A .3 r, T_{3}=\mathrm{A} .3 \mathrm{~m}$. Set $c-\chi_{4}=0$. Then

$$
\beta^{\alpha} \partial_{\alpha}\left[8 a+2 c+2 h_{1}+2 \beta^{\gamma} \partial_{\gamma} n-\beta^{\gamma} a_{7 \gamma}\right]=2 \beta^{\alpha} \beta^{\gamma}\left[\partial_{\alpha} a_{5 \gamma}-y_{5 \alpha \gamma}\right]
$$


(ii) $X=\nabla^{2} N . T_{1}=\mathrm{A} .3 \mathrm{i}, T_{2}=\mathrm{A} .3 \mathrm{j}, T_{3}=\mathrm{A} .3 \mathrm{~d}$. Set $4 p_{4}+8 \rho_{23}=0$. Then

$$
\beta^{\alpha} \partial_{\alpha}\left[8 \rho_{23}+4 c+2 \rho_{12}+2 \beta^{\gamma} \partial_{\gamma} h_{2}-\beta^{\gamma} \rho_{13 \gamma}\right]=2 \beta^{\alpha} \beta^{\gamma}\left[\partial_{\alpha} \rho_{24 \gamma}-x_{2 \alpha \gamma}\right]
$$

(iii) $X=\nabla_{i} N \nabla^{i} N . T_{1}=\mathrm{A} .3 \mathrm{~s}, T_{2}=\mathrm{A} .3 \mathrm{p}, T_{3}=\mathrm{A} .3 \mathrm{t}$. Set $8 p_{3}+4 p_{4}=0$. Then

$$
\beta^{\alpha} \partial_{\alpha}\left[4 \chi_{4}-4 p_{4}+2 \rho_{11}+2 \beta^{\gamma} \partial_{\gamma} \chi_{3}-\beta^{\gamma} \rho_{8 \gamma}\right]=2 \beta^{\alpha} \beta^{\gamma}\left[\partial_{\alpha} y_{\gamma}-x_{\alpha \gamma}\right]
$$

(iv) $X=K \cdot T_{1}=\mathrm{A} .1 \mathrm{f}, T_{2}=\mathrm{A} .1 \mathrm{n}, T_{3}=\mathrm{A} .1 \mathrm{~h}$. Set $j-\rho_{4}=0$. Then

$$
\beta^{\alpha} \partial_{\alpha}\left[4 b+z j+z l_{1}+2 \beta^{\gamma} \partial_{\gamma} m-\beta^{\gamma} \rho_{7 \gamma}\right]=2 \beta^{\alpha} \beta^{\gamma}\left[\partial_{\alpha} b_{8 \gamma}-x_{5 \alpha \gamma}\right]
$$

We have verified that after accounting for differences in basis and notation eq. (5.11) is precisely the same as the general $C$-theorem candidate of this section given in eq. (5.7).

\section{Summary and discussion}

Wess-Zumino consistency conditions for Weyl transformations impose constraints on the renormalization group flow of Weyl anomalies. As a first step in studying these constraints in non-relativistic quantum field theories we have classified the anomalies that appear in $d=2$ (spatial dimensions) at $z=2$ (dynamical exponent at gaussian fixed point). There are many more anomalies than in the comparable relativistic case $(3+1$ dimensions): there are 39 anomalies associated with 4-spatial derivatives (table 2), 6 with 2-time derivatives (table 4) and 32 more that contain 1-time and 2-spatial derivaties (table 5). Freedom to add finite amounts to counterterms gives in turn freedom to shift some anomalies arbitrarily. "Trivial Anomalies" are those that can thus be set to zero. We then classified all counterterms (tables 2-5), gave the shift in Weyl anomalies produced by shifts in counterterms (in appendix B), and then listed the trivial anomalies (table 6).

The consistency conditions among these $39+6+32$ anomalies do not mix among the three sectors. They are listed by sector in appendix A, and from these we can read-off "Vanishing Anomalies" - those that vanish at fixed points; see table. 7. As an application of the use of these conditions we find 6 combinations that give $C$-function candidates. That is, we find (combinations of) anomalies $\tilde{a}$ and $\mathcal{H}_{\alpha \beta}$ that satisfy $\mu d \tilde{a} / d \mu=\mathcal{H}_{\alpha \beta} \beta^{\alpha} \beta^{\beta}$, where $\beta^{\alpha}=\mu d g^{\alpha} / d \mu$ give the flow of the dimensionless coupling constants; then $\tilde{a}$ flows monotonically provided $\mathcal{H}_{\alpha \beta}$ is positive definite. We have not endeavored to attempt to prove that any of our $\mathcal{H}_{\alpha \beta}$ functions are positive definite, and hence our candidates remain just that, candidates. Exploring positivity of these functions in specific examples would be of interest, and determining model-independently under which conditions positivity holds would be more so.

It is important to appreciate the generality, or lack of it thereof, of our results. While we have used some specific form of the Lagrangian in setting up and contextualizing the computation, there is in fact no need to assume this in order to classify the anomalies and compute the consistency conditions. On the other hand we have made a fairly strong 
assumption, that the classical action integral is invariant under the anisotropic scale transformation $\vec{x} \mapsto \lambda \vec{x}, t \mapsto \lambda^{z} t$. All our couplings correspond to marginal deformations. In the $3+1$-dimensional relativistic case relevant deformations do modify the consistency conditions, but the candiate $C$-theorem is not affected, at least by a class of relevant deformations [29]. Clearly, another interesting direction of future study is to investigate the effect of relevant deformations on our consistency conditions: perhaps some of the $6 C$-candidates survive even in the presence of relevant deformations, much as in the relativistic case.

While we have performed a detailed analysis only for the $z=2$ case in $2+1$ dimensions, our results can be readily used in other cases too. For theories in $2+1$ dimensions with $z>0$ and neither $z=2 k$ nor $z=2 / k$ where $k$ is an integer, only the sector of anomalies with 1-time and 2-spatial derivatives remains. Moreover, the classification of anomalies and the consistency conditions for that sector that were derived assuming $z=2$ are valid for arbitrary $z$, with minor modifications in the form of a sprinkling of factors of $z / 2$; we have retained explicit $z$ dependence in the consistency conditions in this sector, eqs. (A.1). This means, in particular, that the $4 C$-candidates in this sector, in eqs. (3.13)-(3.16), are $C$-candidates for arbitrary $z$. For $z=2 k \geq 4$ there are anomalies with $2(k+1)$ spatial derivatives; their classification depends on $z$, so a case-by-case analysis is required. For $z=2 / k \leq 2$ there are anomalies with $k+1$ time derivatives; again their classification depends on $z$ and a case-by-case analysis is required.

For spatial dimensions $d>2$, if $d$ is even a $C$-theorem candidate, in eq. (5.7), becomes available that mimics the one in relativistic theories. Again it relies on assuming only marginal operators are present, but it is possible that, just as in the $3+1$ realtivistic case, the conclusion is not modified by inclusion of relevant deformations. The candidate is based on the anomaly associated with the $d$-dimensional Euler density for the theory on a curved background. Here again it would be interesting to have an explicit example, to test whether the putative metric in coupling constant space, $\mathcal{H}_{\alpha \beta}$, is positive definite. The analysis of a potential $C$-theorem in the case of general dimensions $d$ yields four additional potential $C$-theorems in $d=2$, three for $z=2$ given in eqs. (5.8)-(5.10) and one more for arbitrary $z$, given in eq. (5.11). It deserves mention that all of our proposed $C$ theorem candidates are scheme dependent even at a fixed point. Hence, the value of them at a fixed point can be shifted using counter-terms $F$.

If any of these candidates yields a bona-fide $C$-theorem the presence of limit cycles in non-relativistic quantum field theories is called into question. Limit cycles in relativistic $3+1$ dimensional theories physically correspond to critical points, and the recursive flow corresponds to what amounts to a simultaneous rotation among fundamental fields and marginal operators and their coefficients. Cyclic behavior in non-relativistic quantum systems, on the other hand, do not display continuous scale invariance, so there is no reason to expect that $C$ would remain constant along the flow. The resolution may be that there are no $C$-theorems at all. Or that there are $C$-theorems only under conditions that do not apply to systems that exhibit cycles. We look forward to developments in this area. 


\section{Acknowledgments}

We would like to thank John McGreevy for insightful comments. SP would like to thank Andreas Stergiou for help with Mathematica. This work was supported in part by the US Department of Energy under contract DE- SC0009919.

\section{A Consistency conditions for $2+1 \mathrm{~d}$ NRCFT}

We give below the consistency conditions for the $d=2$ theory. In the $\partial_{t} \nabla^{2}$ sector they are given for arbitrary $z$; else $z=2$ is assumed. The conditions in the $\partial_{t} \nabla^{2}$ sector are as follows:

$$
\begin{aligned}
& \sigma \partial_{t} \sigma^{\prime} \partial_{i} N \partial^{i} g^{\alpha}: \quad-\beta^{\gamma} \partial_{\gamma} \rho_{1 \alpha}-\rho_{1 \gamma} \partial_{\alpha} \beta^{\gamma}+2 \rho_{\alpha}-2 \partial_{\alpha} j+p_{4 \gamma \alpha} \beta^{\gamma}=0 \\
& \sigma \partial_{t} \sigma^{\prime} \nabla^{2} g^{\alpha}: \quad-b_{4 \sigma} \partial_{\alpha} \beta^{\sigma}-\beta^{\sigma} \partial_{\sigma} b_{4 \alpha}+x_{4 \gamma \alpha} \beta^{\gamma}+2 b_{8 \alpha}=0 \\
& \nabla^{2} \sigma \partial_{t} \sigma^{\prime}: \quad 2 k+2 m-z l_{2}-b_{4 \alpha} \beta^{\alpha}+\beta^{\alpha} b_{9 \alpha}=0 \\
& -\sigma^{\prime} \partial_{t} \sigma \nabla^{2} N: 2 j+\beta^{\alpha} \partial_{\alpha} l_{2}=b_{6 \alpha} \beta^{\alpha} \\
& \sigma^{\prime} \partial_{i} \sigma \partial^{i} N \partial_{t} g^{\alpha}: \quad-2 z b_{6 \alpha}-2 z \rho_{6 \alpha}+\rho_{5 \gamma} \partial_{\alpha} \beta^{\gamma}+\beta^{\gamma} \partial_{\gamma} \rho_{5 \alpha}-\beta^{\gamma} p_{4 \alpha \gamma}=0 \\
& \sigma^{\prime} \nabla^{2} \sigma K: \quad 2 b+\beta^{\alpha} \partial_{\alpha} m+z j=\beta^{\alpha} b_{8 \alpha} \\
& \sigma^{\prime} \partial_{t} \sigma R: 2 b-\beta^{\alpha} \partial_{\alpha} k+b_{5 \alpha} \beta^{\alpha}=0 \\
& \sigma^{\prime} \partial_{i} \sigma K \partial^{i} g^{\alpha}: \quad-2 x_{5 \alpha \gamma} \beta^{\gamma}+\beta^{\gamma} \partial_{\gamma} b_{7 \alpha}-z \rho_{\alpha} \\
& +b_{7 \gamma} \partial_{\alpha} \beta^{\gamma}-2 b_{8 \gamma} \partial_{\alpha} \beta^{\gamma}+z \partial_{\alpha} j=0 \\
& \sigma^{\prime} \nabla^{2} \sigma \partial_{t} g^{\alpha}: \quad-x_{4 \alpha \gamma} \beta^{\gamma}+2 b_{5 \alpha}-z b_{6 \alpha}+b_{9 \gamma} \partial_{\alpha} \beta^{\gamma}+\beta^{\gamma} \partial_{\gamma} b_{9 \alpha}=0 \\
& \partial_{i} \sigma \partial_{t} \sigma^{\prime} \partial^{i} g^{\alpha}: \quad-2 b_{4 \gamma} \partial_{\alpha} \beta^{\gamma}+2 b_{7 \alpha}+x_{6 \gamma \alpha} \beta^{\gamma}-2 b_{3 \alpha \gamma} \beta^{\gamma}-z \rho_{1 \alpha}=0 \\
& \sigma \partial_{t} \sigma^{\prime} \partial_{i} g^{\alpha} \partial^{i} g^{\beta}:-\beta^{\gamma} \partial_{\gamma} b_{3 \alpha \beta}-b_{3 \gamma \beta} \partial_{\alpha} \beta^{\gamma} \\
& -b_{3 \gamma \alpha} \partial_{\beta} \beta^{\gamma}-b_{4 \gamma} \partial_{\alpha} \partial_{\beta} \beta^{\gamma}+x_{3 \gamma \alpha \beta} \beta^{\gamma}+2 x_{5 \alpha \beta}=0 \\
& -\sigma \partial_{i} \sigma^{\prime} \partial_{t} g^{\alpha} \partial^{i} g^{\beta}: \quad-2 x_{3 \alpha \gamma \beta} \beta^{\gamma}+x_{6 \sigma \beta} \partial_{\alpha} \beta^{\sigma} \\
& +x_{6 \alpha \gamma} \partial_{\beta} \beta^{\gamma}-2 x_{4 \alpha \sigma} \partial_{\beta} \beta^{\sigma}+\beta^{\gamma} \partial_{\gamma} x_{6 \alpha \beta}-z p_{4 \alpha \beta}=0 \\
& \sigma \partial_{t} \sigma^{\prime} \frac{\partial^{i} N}{N} \frac{\partial_{i} N}{N}: \quad \beta^{\gamma} \partial_{\gamma} \rho_{3}-2 \rho_{4}-\beta^{\alpha} \rho_{6 \alpha}=0 \\
& \sigma \partial_{i} \sigma^{\prime} K \partial^{i} N: 2 z j-\beta^{\alpha} \rho_{\alpha}+\beta^{\alpha} \partial_{\alpha} l_{1}-2 z \rho_{4}=0 \\
& \partial_{i} \sigma \partial_{t} \sigma^{\prime} \partial^{i} N: 2 z \rho_{3}-2 l_{1}+2 z l_{2}+2 j+\rho_{1 \alpha} \beta^{\alpha}=0 \\
& \sigma^{\prime} \partial^{j} \sigma \partial^{i} N\left(K_{i j}-\frac{1}{2} K h_{i j}\right): 2 z f_{1}-\beta^{\alpha} f_{3 \alpha}+z f_{4}-\beta^{\alpha} \partial_{\alpha} f_{7}=0 \\
& \sigma^{\prime} \partial^{j} \sigma \partial^{i} g^{\alpha}\left(K_{i j}-\frac{1}{2} K h_{i j}\right): 2 f_{2 \alpha \gamma} \beta^{\gamma}+z f_{3 \alpha}-\beta^{\gamma} \partial_{\gamma} f_{8 \alpha}-f_{8 \gamma} \partial_{\alpha} \beta^{\gamma}+2 f_{5 \gamma} \partial_{\alpha} \beta^{\gamma}=0 \\
& \sigma^{\prime} \nabla^{i} \partial^{j} \sigma\left(K_{i j}-\frac{1}{2} K h_{i j}\right): \quad z f_{4}+\beta^{\alpha} f_{5 \alpha}-\beta^{\alpha} \partial_{\alpha} f_{6}=0
\end{aligned}
$$

The conditions coming from $\partial_{t}^{2}$ sector are as follows:

$$
\begin{aligned}
\sigma^{\prime} \partial_{t} \sigma K: & 4 d-\beta^{\alpha} \partial_{\alpha} f+\beta^{\alpha} w_{\alpha}=0 \\
\sigma^{\prime} \partial_{t} \sigma \partial_{t} g^{\alpha}: & -2 w_{\alpha}+\beta^{\gamma} \partial_{\gamma} b_{\alpha}+b_{\gamma} \partial_{\alpha} \beta^{\gamma}-2 \chi_{0 \alpha \gamma} \beta^{\gamma}=0
\end{aligned}
$$


The conditions coming from the $\nabla^{4}$ sector are given by:

$$
\begin{aligned}
& \partial_{i} \sigma^{\prime} \nabla^{2} \sigma \partial^{i} g^{\alpha}: \quad-2 \rho_{13 \alpha}-\beta^{\gamma} \rho_{21 \alpha \gamma}+2 a_{7 \alpha} \\
& +2 \chi_{1 \alpha}+2 a_{3 \alpha \gamma} \beta^{\gamma}+2 a_{4 \gamma} \partial_{\alpha} \beta^{\gamma}=0 \\
& \sigma^{\prime} \nabla^{2} \sigma \partial_{i} g^{\alpha} \partial^{i} g^{\beta}: \quad-\beta^{\gamma} t_{2 \gamma \alpha \beta}+\beta^{\gamma} \partial_{\gamma} a_{3 \alpha \beta}+a_{3 \alpha \gamma} \partial_{\beta} \beta^{\gamma} \\
& +a_{3 \beta \gamma} \partial_{\alpha} \beta^{\gamma}+a_{4 \gamma} \partial_{\alpha} \partial_{\beta} \beta^{\gamma}+2 y_{5 \alpha \beta}-2 x_{2 \alpha \beta}=0 \\
& \sigma^{\prime} \nabla^{2} \sigma \nabla^{2} g^{\alpha}: \quad 2 a_{5 \alpha}+\beta^{\gamma} \partial_{\gamma} a_{4 \alpha}+a_{4 \gamma} \partial_{\alpha} \beta^{\gamma}-2 \rho_{22 \alpha \gamma} \beta^{\gamma}-2 \rho_{24 \alpha}=0 \\
& \sigma^{\prime} \partial_{i} \sigma \partial^{i} g^{\alpha} \nabla^{2} N: \quad-2 x_{2 \alpha \gamma} \beta^{\gamma}+\beta^{\gamma} \partial_{\gamma} \rho_{13 \alpha}+\rho_{13 \gamma} \partial_{\alpha} \beta^{\gamma} \\
& -2 \rho_{24 \gamma} \partial_{\alpha} \beta^{\gamma}-2 \rho_{25 \alpha}=0 \\
& \sigma^{\prime} \partial_{i} \sigma \partial^{i} N \partial_{j} N \partial^{j} g^{\alpha}: \quad-2 p_{5 \beta \alpha} \beta^{\beta}+\beta^{\gamma} \partial_{\gamma} \rho_{10 \alpha}+\rho_{10 \gamma} \partial_{\alpha} \beta^{\gamma}-4 \rho_{25 \alpha}-4 \rho_{9 \alpha}=0 \\
& \sigma^{\prime} \partial_{i} \sigma \partial^{i} g^{\alpha} \partial_{j} g^{\beta} \partial^{j} g^{\gamma}: \quad-4 x_{\alpha \sigma \beta \gamma} \beta^{\sigma}+\beta^{\sigma} \partial_{\sigma} t_{\alpha \beta \gamma}+t_{\sigma \beta \gamma} \partial_{\alpha} \beta^{\sigma}+t_{\alpha \sigma \gamma} \partial_{\beta} \beta^{\sigma}+t_{\alpha \sigma \beta} \partial_{\gamma} \beta^{\sigma} \\
& -2 t_{2 \sigma \beta \gamma} \partial_{\alpha} \beta^{\sigma}+\rho_{21 \alpha \sigma} \partial_{\beta} \partial_{\gamma} \beta^{\sigma}-2 x_{\alpha \beta \gamma}=0 \\
& \sigma^{\prime} \partial_{i} \sigma \partial^{i} g^{\alpha} \partial_{j} N \partial^{j} g^{\beta}: \quad-4 p_{5 \alpha \beta}-2 \rho_{26 \beta \gamma} \partial_{\alpha} \beta^{\gamma} \\
& +x_{1 \alpha \gamma} \partial_{\beta} \beta^{\gamma}+x_{1 \gamma \beta} \partial_{\alpha} \beta^{\gamma}+\beta^{\gamma} \partial_{\gamma} x_{1 \alpha \beta}-2 x_{\alpha \gamma \beta} \beta^{\gamma}=0 \\
& \sigma \partial_{i} \sigma^{\prime} \partial^{i} g^{\alpha} \nabla^{2} g^{\beta}: \quad-\beta^{\gamma} \partial_{\gamma} \rho_{21 \alpha \beta}-\rho_{21 \gamma \beta} \partial_{\alpha} \beta^{\gamma}-\rho_{21 \alpha \gamma} \partial_{\beta} \beta^{\gamma} \\
& +4 \rho_{22 \gamma \beta} \partial_{\alpha} \beta^{\gamma}+2 \rho_{26 \alpha \beta}+2 t_{2 \beta \gamma \alpha} \beta^{\gamma}=0 \\
& \sigma^{\prime} \nabla^{2} \sigma \nabla^{2} N: \quad-4 \rho_{23}-\beta^{\gamma} \rho_{24 \gamma}+\beta^{\gamma} \partial_{\gamma} h_{2}-2 c=0 \\
& \sigma^{\prime} \partial_{i} \sigma \partial^{i} N \nabla^{2} N: \quad 4 p_{4}-\beta^{\alpha} \partial_{\alpha} \rho_{12}+8 \rho_{23}+\beta^{\gamma} \rho_{25 \gamma}=0 \\
& \sigma^{\prime} \partial_{i} \sigma \partial^{i} N \nabla^{2} g^{\alpha}: \quad-4 y_{\alpha}+\beta^{\gamma} \partial_{\gamma} \rho_{7 \alpha}+\rho_{7 \gamma} \partial_{\alpha} \beta^{\gamma}-4 \rho_{24 \alpha}-\beta^{\gamma} \rho_{26 \gamma \alpha}=0 \\
& -\sigma^{\prime} \nabla^{2} \sigma R: \quad-a_{5 \alpha} \beta^{\alpha}+4 a+2 c+\beta^{\alpha} \partial_{\alpha} n=0 \\
& \sigma^{\prime} \partial_{i} \sigma R \partial^{i} g^{\alpha}:-2 y_{5 \alpha \gamma} \beta^{\gamma}-2 \chi_{\alpha}+2 \partial_{\alpha} c \\
& +\beta^{\gamma} \partial_{\gamma} a_{7 \alpha}+a_{7 \gamma} \partial_{\alpha} \beta^{\gamma}-2 a_{5 \gamma} \partial_{\alpha} \beta^{\gamma}=0 \\
& \sigma \nabla^{2} \sigma^{\prime} \partial^{i} g^{\alpha} \partial_{i} N: \quad-\beta^{\gamma} \partial_{\gamma} \chi_{1 \alpha}-\chi_{1 \gamma} \partial_{\alpha} \beta^{\gamma}+2 \partial_{\alpha} c-2 \chi_{\alpha}+\rho_{26 \alpha \gamma} \beta^{\gamma}+2 \rho_{25 \alpha}=0 \\
& \partial_{i} \sigma \nabla^{2} \sigma^{\prime} \partial^{i} N: \quad 2 h_{1}+4 h_{2}-2 c+\beta^{\alpha} \chi_{1 \alpha}+4 \chi_{3}-\beta^{\alpha} \rho_{7 \alpha}-2 \rho_{12}=0 \\
& \sigma^{\prime} \partial_{i} \sigma \partial^{i} N \partial_{j} N \partial^{j} N: \quad 8 p_{3}-\beta^{\alpha} \partial_{\alpha} \rho_{11}+\rho_{9 \alpha} \beta^{\alpha}+4 p_{4}=0 \\
& \sigma \partial_{i} \sigma^{\prime} \partial_{j} g^{\alpha} \partial^{j} g^{\beta} \partial^{i} N: \quad 4 x_{\alpha \beta}-\rho_{7 \gamma} \partial_{\beta} \partial_{\alpha} \beta^{\gamma}-\rho_{1 \gamma \beta} \partial_{\alpha} \beta^{\gamma} \\
& -\rho_{1 \gamma \alpha} \partial_{\beta} \beta^{\gamma}-\beta^{\gamma} \partial_{\gamma} \rho_{1 \alpha \beta}+\beta^{\gamma} x_{\gamma \alpha \beta}+4 x_{2 \alpha \beta}=0 \\
& \sigma \partial_{i} \sigma^{\prime} R \partial^{i} N: \quad 4 c+\beta^{\alpha} \partial_{\alpha} h_{1}-\beta^{\alpha} \chi_{\alpha}-4 \chi_{4}=0 \\
& \sigma^{\prime} \nabla^{2} \sigma \partial^{i} N \partial_{i} N: \quad 2 \chi_{4}+\beta^{\alpha} \partial_{\alpha} \chi_{3}-\beta^{\alpha} y_{\alpha}-2 p_{4}=0 \\
& \sigma^{\prime} \partial_{i} \sigma \partial_{j} N \partial^{j} N \partial^{i} g^{\alpha}: \quad-2 x_{\alpha \gamma} \beta^{\gamma}+\beta^{\gamma} \partial_{\gamma} \rho_{8 \alpha}+\rho_{8 \gamma} \partial_{\alpha} \beta^{\gamma}-2 \rho_{9 \alpha}-2 y_{\gamma} \partial_{\alpha} \beta^{\gamma}=0
\end{aligned}
$$

\section{B Anomaly ambiguities}

As explained in section 3.2 the freedom to shift counter-terms by finite amount makes anomaly coefficients ambiguous. We list here the precise form of these ambiguities, in the $\partial_{t} \nabla^{2}$ sector they are given for arbitrary $z$; else $z=2$ is assumed: 


\section{B.1 $\partial_{t}^{2}$ sector}

$$
\begin{aligned}
\delta f & =-4 D-\beta^{\alpha} W_{\alpha} \\
\delta w_{\alpha} & =-\left[\beta^{\gamma} \partial_{\gamma} W_{\alpha}+W_{\gamma} \partial_{\alpha} \beta^{\gamma}\right] \\
\delta b_{\alpha} & =-2 W_{\alpha}-2 X_{0 \alpha \gamma} \beta^{\gamma} \\
\delta \chi_{0 \alpha \beta} & =-\beta^{\gamma} \partial_{\gamma} X_{0 \alpha \beta}-X_{0 \alpha \gamma} \partial_{\beta} \beta^{\gamma}-X_{0 \alpha \gamma} \partial_{\beta} \beta^{\gamma} \\
\delta d & =-\beta^{\alpha} \partial_{\alpha} D \\
\delta e & =-\beta^{\alpha} \partial_{\alpha} E
\end{aligned}
$$

\section{B.2 $\partial_{t} \nabla^{2}$ sector}

$$
\begin{aligned}
& \delta \rho_{4}=-\beta^{\alpha} \partial_{\alpha} P \\
& \delta x_{5 \alpha \beta}=-P_{3 \gamma} \partial_{\alpha} \partial_{\beta} \beta^{\gamma}-\beta^{\gamma} \partial_{\gamma} X_{5 \alpha \beta}-X_{5 \gamma \beta} \partial_{\alpha} \beta^{\gamma}-X_{5 \gamma \alpha} \partial_{\beta} \beta^{\gamma} \\
& \delta \rho_{\alpha}=-\beta^{\gamma} \partial_{\gamma} P_{\alpha}-P_{\gamma} \partial_{\alpha} \beta^{\gamma} \\
& \delta j=-\beta^{\alpha} \partial_{\alpha} L \\
& \delta b_{8 \alpha}=-\beta^{\gamma} \partial_{\gamma} P_{3 \alpha}-P_{3 \gamma} \partial_{\alpha} \beta^{\gamma} \\
& \delta b=-\beta^{\alpha} \partial_{\alpha} B \\
& \delta m=2 B+z L-P_{3 \alpha} \beta^{\alpha} \\
& \delta l_{1}=-2 z P+2 z L-\beta^{\alpha} P_{\alpha} \\
& \delta b_{7 \alpha}=-2 P_{3 \gamma} \partial_{\alpha} \beta^{\gamma}+z \partial_{\alpha} L-z P_{\alpha}-2 X_{5 \alpha \gamma} \beta^{\gamma} \\
& \delta \rho_{6 \alpha}=-X_{\gamma} \partial_{\alpha} \beta^{\gamma}-\beta^{\gamma} \partial_{\gamma} X_{\alpha} \\
& \delta x_{3 \alpha \beta \gamma}=-\beta^{\sigma} \partial_{\sigma} X_{3 \alpha \beta \gamma}-X_{3 \sigma \beta \gamma} \partial_{\alpha} \beta^{\sigma}-X_{3 \alpha \sigma \gamma} \partial_{\beta} \beta^{\sigma}-X_{3 \alpha \sigma \beta} \partial_{\gamma} \beta^{\sigma}-X_{4 \alpha \sigma} \partial_{\gamma} \partial_{\beta} \beta^{\sigma} \\
& \delta p_{4 \alpha \beta}=-\beta^{\gamma} \partial_{\gamma} P_{4 \alpha \beta}-P_{4 \gamma \beta} \partial_{\alpha} \beta^{\gamma}-P_{4 \alpha \gamma} \partial_{\beta} \beta^{\gamma} \\
& \delta b_{6 \alpha}=-\beta^{\gamma} \partial_{\gamma} B_{6 \alpha}-B_{6 \gamma} \partial_{\alpha} \beta^{\gamma} \\
& \delta x_{4 \alpha \beta}=-X_{4 \gamma \beta} \partial_{\alpha} \beta^{\gamma}-\beta^{\gamma} \partial_{\gamma} X_{4 \alpha \beta}-X_{4 \alpha \gamma} \partial_{\beta} \beta^{\gamma} \\
& \delta b_{5 \alpha}=-\beta^{\gamma} \partial_{\gamma} B_{5 \alpha}-B_{5 \gamma} \partial_{\alpha} \beta^{\gamma} \\
& \delta b_{9 \alpha}=2 B_{5 \alpha}-z B_{6 \alpha}-\beta^{\gamma} X_{4 \alpha \gamma} \\
& \delta \rho_{5 \alpha}=-2 z X_{\alpha}-2 z B_{6 \alpha}-P_{4 \alpha \gamma} \beta^{\gamma} \\
& \delta x_{6 \alpha \beta}=-X_{4 \alpha \gamma} \partial_{\beta} \beta^{\gamma}-2 X_{3 \alpha \gamma \beta} \beta^{\gamma}-z P_{4 \alpha \beta} \\
& \delta \rho_{3}=-2 P-\beta^{\alpha} X_{\alpha} \\
& \delta b_{3 \alpha \beta}=-2 X_{5 \alpha \beta}-X_{3 \gamma \beta \alpha} \beta^{\gamma} \\
& \delta \rho_{1 \alpha}=-2 P_{\alpha}+\partial_{\alpha} 2 L-P_{4 \gamma \alpha} \beta^{\gamma} \\
& \delta l_{2}=2 L-B_{6 \alpha} \beta^{\alpha} \\
& \delta b_{4 \alpha}=-2 P_{3 \alpha}-X_{4 \gamma \alpha} \beta^{\gamma} \\
& \delta k=-2 B-B_{5 \alpha} \beta^{\alpha} \\
& \delta f_{1}=-\beta^{\alpha} \partial_{\alpha} F_{1} \\
& \delta f_{2 \alpha \beta}=-\beta^{\gamma} \partial_{\gamma} F_{2 \alpha \beta}-F_{2 \gamma \beta} \partial_{\alpha} \beta^{\gamma}-F_{2 \alpha \gamma} \partial_{\beta} \beta^{\gamma}-F_{5 \gamma} \partial_{\alpha} \partial_{\beta} \beta^{\gamma}
\end{aligned}
$$




$$
\begin{aligned}
\delta f_{3 \alpha} & =-\beta^{\gamma} \partial_{\gamma} F_{3 \alpha}-F_{3 \gamma} \partial_{\alpha} \beta^{\gamma} \\
\delta f_{4} & =-\beta^{\gamma} \partial_{\gamma} F_{4} \\
\delta f_{5 \alpha} & =-\beta^{\gamma} \partial_{\gamma} F_{5 \alpha}-F_{5 \gamma} \partial_{\alpha} \beta^{\gamma} \\
\delta f_{6} & =-z F_{4}-F_{5 \alpha} \beta^{\alpha} \\
\delta f_{7} & =-2 z F_{1}-F_{3 \alpha} \beta^{\alpha}-z F_{4} \\
\delta f_{8 \alpha} & =-z F_{3 \alpha}-2 F_{2 \gamma \alpha} \beta^{\gamma}-2 F_{5 \gamma} \partial_{\alpha} \beta^{\gamma}
\end{aligned}
$$

\section{B.3 $\nabla^{4}$ sector}

$$
\begin{aligned}
\delta p_{3} & =-\beta^{\alpha} \partial_{\alpha} P_{3} \\
\delta x_{\alpha \beta} & =-Y_{\gamma} \partial_{\beta} \partial_{\alpha} \beta^{\gamma}-X_{\gamma \beta} \partial_{\alpha} \beta^{\gamma}-X_{\gamma \alpha} \partial_{\beta} \beta^{\gamma}-\beta^{\gamma} \partial_{\gamma} X_{\alpha \beta} \\
\delta \rho_{9 \alpha} & =-\beta^{\gamma} \partial_{\gamma} P_{1 \alpha}-P_{1 \gamma} \partial_{\alpha} \beta^{\gamma} \\
\delta p_{4} & =-\beta^{\alpha} \partial_{\alpha} P_{4} \\
\delta y_{\alpha} & =-\beta^{\gamma} \partial_{\gamma} Y_{\alpha}-Y_{\gamma} \partial_{\alpha} \beta^{\gamma} \\
\delta \chi_{4} & =-\beta^{\alpha} \partial_{\alpha} Q \\
\delta \chi_{3} & =2 Q-\beta^{\alpha} Y_{\alpha}-2 P_{4} \\
\delta \rho_{11} & =-8 P_{3}-P_{1 \alpha} \beta^{\alpha}-4 P_{4} \\
\delta \rho_{8 \alpha} & =-2 P_{1 \alpha}-2 X_{\alpha \gamma} \beta^{\gamma}-2 Y_{\gamma} \partial_{\alpha} \beta^{\gamma} \\
\delta x_{\alpha \beta \gamma \delta} & =-\beta^{\sigma} \partial_{\sigma} X_{\alpha \beta \gamma \delta}-X_{\sigma \beta \gamma \delta} \partial_{\alpha} \beta^{\sigma}-X_{\alpha \sigma \gamma \delta} \partial_{\beta} \beta^{\sigma}-X_{\alpha \beta \sigma \delta} \partial_{\gamma} \beta^{\sigma} \\
& =X_{\alpha \beta \gamma \sigma} \partial_{\gamma} \beta^{\sigma}-T_{2 \sigma \alpha \beta} \partial_{\delta} \partial_{\gamma} \beta^{\sigma} \\
\delta x_{\alpha \beta \gamma} & =-\beta^{\sigma} \partial_{\sigma} X_{\alpha \beta \gamma}-X_{\sigma \beta \gamma} \partial_{\alpha} \beta^{\sigma}-X_{\alpha \sigma \gamma} \partial_{\beta} \beta^{\sigma}-X_{\alpha \beta \sigma} \partial_{\gamma} \beta^{\sigma}-P_{26 \alpha \sigma} \partial_{\gamma} \partial_{\beta} \beta^{\sigma} \\
\delta x_{2 \alpha \beta} & =-X_{2 \gamma \beta} \partial_{\alpha} \beta^{\gamma}-X_{2 \gamma \alpha} \partial_{\beta} \beta^{\gamma}-\beta^{\gamma} \partial_{\gamma} X_{2 \alpha \beta}-P_{24 \gamma} \partial_{\alpha} \partial_{\beta} \beta^{\gamma} \\
\delta t_{2 \alpha \beta \gamma} & =-\beta^{\sigma} \partial_{\sigma} T_{2 \alpha \beta \gamma}-T_{2 \sigma \beta \gamma} \partial_{\alpha} \beta^{\sigma}-T_{2 \alpha \sigma \gamma} \partial_{\beta} \beta^{\sigma}-T_{2 \alpha \beta \sigma} \partial_{\gamma} \beta^{\sigma}-2 P_{22 \sigma \alpha} \partial_{\gamma} \partial_{\beta} \beta^{\sigma} \\
\delta y_{5 \alpha \beta} & =-A_{5 \gamma} \partial_{\alpha} \partial_{\beta} \beta^{\gamma}-Y_{5 \alpha \gamma} \partial_{\beta} \beta^{\gamma}-Y_{5 \beta \gamma} \partial_{\alpha} \beta^{\gamma}-\beta^{\gamma} \partial_{\gamma} Y_{5 \alpha \beta} \\
\delta a_{3 \alpha \beta} & =-2 X_{2 \alpha \beta}-\beta^{\gamma} T_{2 \gamma \alpha \beta}+2 Y_{5 \alpha \beta} \\
\delta \rho_{1 \alpha \beta} & =-4 X_{\alpha \beta}-X_{\gamma \alpha \beta} \beta^{\gamma}-4 X_{2 \alpha \beta} \\
\delta t_{\alpha \beta \gamma} & =-4 X_{\alpha \sigma \beta \gamma} \beta^{\sigma}-2 T_{2 \sigma \gamma \beta} \partial_{\alpha} \beta^{\sigma}-2 X_{\alpha \beta \gamma} \\
\delta p_{5 \alpha \beta} & =-\beta^{\gamma} \partial_{\gamma} P_{5 \alpha \beta}-P_{5 \gamma \beta} \partial_{\alpha} \beta^{\gamma}-P_{5 \gamma \alpha} \partial_{\beta} \beta^{\gamma} \\
\delta \rho_{25 \alpha} & =-\beta^{\gamma} \partial_{\gamma} P_{25 \alpha}-P_{25 \gamma} \partial_{\alpha} \beta^{\gamma} \\
\delta \rho_{26 \alpha \beta} & =-\beta^{\gamma} \partial_{\gamma} P_{26 \alpha \beta}-P_{26 \gamma \beta} \partial_{\alpha} \beta^{\gamma}-P_{26 \alpha \gamma} \partial_{\beta} \beta^{\gamma} \\
\delta \chi_{\alpha} & =-\beta^{\gamma} \partial_{\gamma} Q_{\alpha}-Q_{\gamma} \partial_{\alpha} \beta^{\gamma} \\
\delta \chi_{1 \alpha} & =2 Q_{\alpha}-2 \partial_{\alpha} H-2 P_{25 \alpha}-\beta^{\gamma} P_{26 \alpha \gamma} \\
\delta \rho_{10 \alpha} & =-4 P_{1 \alpha}-4 P_{25 \alpha}-2 P_{5 \gamma \alpha} \beta^{\gamma} \\
\delta x_{1 \alpha \beta} & =-4 P_{5 \alpha \beta}-2 X_{\alpha \gamma \beta} \beta^{\gamma}-2 P_{26 \beta \gamma} \partial_{\alpha} \beta^{\gamma} \\
\delta \rho_{23} & =-\beta^{\gamma} \partial_{\gamma} P_{23} \\
\delta \rho_{24 \alpha} & =-\beta^{\gamma} \partial_{\gamma} P_{24 \alpha}-P_{24 \gamma} \partial_{\alpha} \beta^{\gamma} \\
\delta c & =-\beta^{\alpha} \partial_{\alpha} H \\
\delta & \\
\delta &
\end{aligned}
$$




$$
\begin{aligned}
\delta h_{2} & =-2 H-4 P_{23}-\beta^{\gamma} P_{24 \gamma} \\
\delta \rho_{12} & =-4 P_{4}-8 P_{23}-P_{25 \alpha} \beta^{\alpha} \\
\delta \rho_{13 \alpha} & =-2 P_{24 \gamma} \partial_{\alpha} \beta^{\gamma}-2 P_{25 \alpha}-2 \beta^{\gamma} X_{2 \alpha \gamma} \\
\delta \rho_{22 \alpha \beta} & =-\beta^{\gamma} \partial_{\gamma} P_{22 \alpha \beta}-P_{22 \alpha \gamma} \partial_{\beta} \beta^{\gamma}-P_{22 \gamma \beta} \partial_{\alpha} \beta^{\gamma} \\
\delta a_{5 \alpha} & =-\beta^{\gamma} \partial_{\gamma} A_{5 \alpha}-A_{5 \gamma} \partial_{\alpha} \beta^{\gamma} \\
\delta a_{4 \alpha} & =2 A_{5 \alpha}-2 \beta^{\gamma} P_{22 \gamma \alpha}-2 P_{24 \alpha} \\
\delta \rho_{7 \alpha} & =-4 Y_{\alpha}-4 P_{24 \alpha}-P_{26 \gamma \alpha} \beta^{\gamma} \\
\delta \rho_{21 \alpha \beta} & =-2 T_{2 \alpha \gamma \beta} \beta^{\gamma}-4 P_{22 \gamma \beta} \partial_{\alpha} \beta^{\gamma}-2 P_{26 \alpha \beta} \\
\delta a & =-\beta^{\alpha} \partial_{\alpha} A \\
\delta n & =4 A+2 H-A_{5 \alpha} \beta^{\alpha} \\
\delta h_{1} & =4 H-4 Q-\beta^{\alpha} Q_{1 \alpha} \\
\delta a_{7 \alpha} & =-2 A_{5 \gamma} \partial_{\alpha} \beta^{\gamma}-2 Y_{5 \alpha \gamma} \beta^{\gamma}-2 Q_{\alpha}+2 \partial_{\alpha} H
\end{aligned}
$$

\section{S-theorem: $0+1 \mathrm{D}$ conformal quantum mechanics}

One may wonder whether the formalism that leads to the Weyl anomaly and consistency conditions can be used for the case of $d=0$. One encounters an immediate obstacle when attempting this. There is no immediate generalization of the trace anomaly equation (2.22). The problem is that there is no extension of the action integral that gives invariance under the local version of rescaling transformations, because there is no extrinsic curvature tensor at our disposal. The naive generalisation of the Callan-Symanzik equation specialized to $d=0, H=\beta^{\alpha} \mathcal{O}_{\alpha}$, cannot hold. In fact, for example, the free particle is a scale invariant system with $H \neq 0$.

The inverse square potential serves as a test ground for a simple realisation of the quantum anomaly, where the classical scale symmetry is broken by quantum mechanical effects [62] leading to dimensional transmutation i.e, after renormalization the quantum system acquires an intrinsic length scale [63, 64]. Studies have been made of non-selfadjointness of the Hamiltonian in the strongly attractive regime and how to obtain its selfadjoint extension, a procedure that effectively amounts to renormalisation $[65,66]$. The system is also shown to exhibit limit cycle behaviour in renormalization group flows [67, 68]. This potential appears in different branches of physics, from nuclear physics $[68,69]$ and molecular physics [70] to quantum cosmology [71-73] and the study of black holes [74]. Given this, it is of interest to understand how quantum effects break scale symmetry in non-relativist quantum mechanics. We will prove a general theorem concerning the breaking of scale symmetry.

In the quantum mechanical description of a scale invariant system, the Hamiltonian $H$ and the generator of scale transformations $D$ obey the following commutation relation:

$$
[D, H]=i z H
$$

where $z$ is the dynamical exponent of the theory. We will show an elementary $S$-Theorem, that (C.1) is incompatible with $H$ being Hermitian on a domain containing the state ${ }^{4} D|E\rangle$,

\footnotetext{
${ }^{4}$ That is, the action of $D$ on non-zero energy eigenstates is well defined.
} 
where $|E\rangle$ is any non-zero energy eigenstate. The $S$-Theorem can be used to deduce that classically scale invariant systems, e.g., the inverse square potential, cannot be quantized without loosing either unitarity or scale invariance if we insist on having bound states with finite non-zero binding energy.

To prove the theorem, we consider the eigenstates $|E\rangle$ of the Hamiltonian $H$ and take expectation value of the $[D, H]$ in these eigenstates. We have

$$
\langle E|[D, H]| E\rangle=\langle E|D H| E\rangle-\langle E|H D| E\rangle
$$

Assuming $H$ is hermitian and $D$ is well defined we have

$$
\langle E|[D, H]| E\rangle=0
$$

On the other hand, scale invariance, eq. (C.1), implies

$$
\langle E|[D, H]| E\rangle=i z\langle E|H| E\rangle \neq 0
$$

Comparing (C.3) and (C.4), proves the theorem. It deserves mentioning that the mismatch is not due to the real part of the quantity $\langle E|[D, H]| E\rangle$ since,

$$
\operatorname{Re}(\langle E|[D, H]| E\rangle)=0
$$

is consistent with

$$
\operatorname{Re}(\langle E|i z H| E\rangle)=0
$$

That the mismatch between (C.3) and (C.4) lies in the imaginary part hints at the fact that $H$ can not be hermitian if we have scale invariance. We recall that hermiticity of $H$ crucially depends on vanishing of a boundary term, which is imaginary when we consider quantities like $\langle E|H| E\rangle$.

For a simple illustration of $S$-theorem consider the free particle with one degree of freedom, $H=\frac{1}{2} p^{2}$ and $D=\frac{1}{2}(x p+p x)-t H$. Consider first the particle in a finite periodic box with length $L$. The operator algebra of the free particle holds regardless of the presence of the periodic boundaries, so the $S$-theorem holds and it tells us that either $H$ is not hermitian or $D|p\rangle$ is not a state. It is instructive to look carefully at the derivation of (C.3) and (C.4) in this context. An elementary computation gives

$$
\langle p|(H D|p\rangle)-\langle p|(D H|p\rangle)=-i p^{2}
$$

which is consistent with the scaling algebra

$$
[D, H]=2 i H
$$

but consistency comes at the expense of rendering $H$ non-hermitian on a domain which contains the state $D|p\rangle$. Indeed, for the periodic box $D|p\rangle$ does not belong in the Hilbert space since $\langle x|D| p\rangle$ is not periodic. Hence, the apparent loss of hermiticity is irrelevant as it involves only functions that are not states. In the boundary free case $(L \rightarrow \infty)$ the normalization of the continuum of energy eigenfunctions is by a Dirac-delta distribution, 
and the norm of the functions $\langle x|D| p\rangle$ involves up to two derivatives of the distribution. If we include these functions in the Hilbert space the Hamiltonian is not hermitian. On the other hand, if we choose the Hilbert space to be that of square integrable functions, then $H$ is hermitian but neither $\langle x \mid p\rangle$ nor $\langle x|D| p\rangle$ are in the Hilbert space.

In contrast, consider the inverse square potential problem. For sufficiently strong attractive potential there are normalizable bound states $|E\rangle$, and the state $D|E\rangle$ is properly normalized. The Hamiltonian is hermitian, but this case requires reguralisation and renormalization and scale symmetry is broken.

This is in fact a statement of a more general result. A corollary of the $S$-theorem is that we cannot have (properly normalized) bound states with non-zero energy in a scale invariant system if we insist on the Hamiltonian being hermitian on the Hilbert space. As in the previous example, this follows from observing that if there exists a properly normalized state $|E\rangle$, then $D|E\rangle$ is also a properly normalized state since the wave-function vanishes sufficiently fast at infinity. This result is consistent with representation theory: a discrete spectrum $\left\{E_{n}\right\}$ cannot form a representation of a transformation which acts by $E \rightarrow \lambda^{z} E$ for continuous $\lambda$, (except if the only allowed finite energy value is $E=0$ ). For example, it is well known that for the inverse square problem in the strongly attractive regime, continuous spectrum is an illusion since in that regime, $H$ is no more Hermitian. To make $H$ hermitian, we need to renormalize the problem, breaking the scale symmetry.

The $S$-theorem can be generalised to to any Hermitian operator $A$ with non zero scaling dimension $\alpha$, that is, $[D, A]=i \alpha A$. The operator $A$ can not be Hermitian on a domain containing $D|A\rangle$ where $|A\rangle$ is the eigenstate of operator $A$. In particular, if we want $A$ to be hermitian on a Hilbert space, $\mathcal{L}^{2}$, then the state $D|A\rangle$ can not belong to $\mathcal{L}^{2}$. For example, $A$ can be the momentum operator $p$, which is hermitian on a rigged Hilbert space and has a non-zero scaling dimension. This generalized $S$-theorem implies that $D|p\rangle$ can not belong to the rigged Hilbert space, which is indeed the case.

Open Access. This article is distributed under the terms of the Creative Commons Attribution License (CC-BY 4.0), which permits any use, distribution and reproduction in any medium, provided the original author(s) and source are credited.

\section{References}

[1] C.A. Regal, M. Greiner and D.S. Jin, Observation of Resonance Condensation of Fermionic Atom Pairs, Phys. Rev. Lett. 92 (2004) 040403 [INSPIRE].

[2] M.W. Zwierlein, C.A. Stan, C.H. Schunck, S.M.F. Raupach, A.J. Kerman and W. Ketterle, Condensation of Pairs of Fermionic Atoms near a Feshbach Resonance, Phys. Rev. Lett. 92 (2004) 120403 [INSPIRE].

[3] D.M. Eagles, Possible Pairing without Superconductivity at Low Carrier Concentrations in Bulk and Thin-Film Superconducting Semiconductors, Phys. Rev. 186 (1969) 456 [INSPIRE].

[4] A. Leggett, Diatomic molecules and cooper pairs, in Modern Trends in the Theory of Condensed Matter, Lect. Notes Phys. 115 (1980) 13.

[5] A.J. Leggett, Cooper Pairing In Spin-Polarized Fermi Systems, J. Phys. Colloques 41 (1980) C7. 
[6] P. Nozieres and S. Schmitt-Rink, Bose condensation in an attractive fermion gas: From weak to strong coupling superconduct ivity, J. Low. Temp. Phys. 59 (1985) 195 [inSPIRE].

[7] D.B. Kaplan, M.J. Savage and M.B. Wise, A new expansion for nucleon-nucleon interactions, Phys. Lett. B 424 (1998) 390 [nucl-th/9801034] [INSPIRE].

[8] D.B. Kaplan, M.J. Savage and M.B. Wise, Two nucleon systems from effective field theory, Nucl. Phys. B 534 (1998) 329 [nucl-th/9802075] [inSPIRE].

[9] J.L. Roberts, N.R. Claussen, J.P. Burke, C.H. Greene, E.A. Cornell and C.E. Wieman, Resonant Magnetic Field Control of Elastic Scattering in Cold R-85b, Phys. Rev. Lett. 81 (1998) 5109 [INSPIRE].

[10] C. Chin, V. Vuletić, A.J. Kerman and S. Chu, High Resolution Feshbach Spectroscopy of Cesium, Phys. Rev. Lett. 85 (2000) 2717.

[11] P.J. Leo, C.J. Williams and P.S. Julienne, Collision Properties of Ultracold ${ }^{133}$ Cs Atoms, Phys. Rev. Lett. 85 (2000) 2721.

[12] T. Loftus, C.A. Regal, C. Ticknor, J.L. Bohn and D.S. Jin, Resonant Control of Elastic Collisions in an Optically Trapped Fermi Gas of Atoms, Phys. Rev. Lett. 88 (2002) 173201.

[13] T.D. Lee and G.C. Wick, Negative Metric and the Unitarity of the S Matrix, Nucl. Phys. B 9 (1969) 209 [INSPIRE].

[14] T.D. Lee and G.C. Wick, Finite Theory of Quantum Electrodynamics, Phys. Rev. D 2 (1970) 1033 [INSPIRE].

[15] B. Grinstein, D. O'Connell and M.B. Wise, The Lee-Wick standard model, Phys. Rev. D 77 (2008) 025012 [arXiv:0704.1845] [INSPIRE].

[16] B. Grinstein, D. O'Connell and M.B. Wise, Causality as an emergent macroscopic phenomenon: The Lee-Wick O(N) model, Phys. Rev. D 79 (2009) 105019 [arXiv:0805.2156] [INSPIRE].

[17] S.W. Hawking and T. Hertog, Living with ghosts, Phys. Rev. D 65 (2002) 103515 [hep-th/0107088] [INSPIRE].

[18] P. Hořava, Quantum Gravity at a Lifshitz Point, Phys. Rev. D 79 (2009) 084008 [arXiv: 0901.3775] [INSPIRE].

[19] D. Anselmi and M. Halat, Renormalization of Lorentz violating theories, Phys. Rev. D 76 (2007) 125011 [arXiv:0707.2480] [inSPIRE].

[20] D. Anselmi, Weighted scale invariant quantum field theories, JHEP 02 (2008) 051 [arXiv:0801.1216] [INSPIRE].

[21] D. Anselmi, Weighted power counting and Lorentz violating gauge theories. I. General properties, Annals Phys. 324 (2009) 874 [arXiv:0808.3470] [INSPIRE].

[22] D. Anselmi, Weighted power counting and Lorentz violating gauge theories. II. Classification, Annals Phys. 324 (2009) 1058 [arXiv:0808.3474] [INSPIRE].

[23] M. Baggio, J. de Boer and K. Holsheimer, Anomalous Breaking of Anisotropic Scaling Symmetry in the Quantum Lifshitz Model, JHEP 07 (2012) 099 [arXiv:1112.6416] [INSPIRE].

[24] I. Arav, S. Chapman and Y. Oz, Lifshitz Scale Anomalies, JHEP 02 (2015) 078 [arXiv: 1410.5831] [INSPIRE]. 
[25] I. Arav, S. Chapman and Y. Oz, Relaxation of nonspherical sessile drops towards equilibrium, Phys. Rev. E 65 (2002) 046135 [arXiv: 1601.06957].

[26] R. Auzzi, S. Baiguera and G. Nardelli, On Newton-Cartan trace anomalies, JHEP 02 (2016) 003 [Erratum ibid. 1602 (2016) 177] [arXiv:1511.08150] [INSPIRE].

[27] K. Jensen, Anomalies for Galilean fields, arXiv:1412.7750 [INSPIRE].

[28] T. Griffin, P. Hořava and C.M. Melby-Thompson, Conformal Lifshitz Gravity from Holography, JHEP 05 (2012) 010 [arXiv:1112.5660] [INSPIRE].

[29] H. Osborn, Weyl consistency conditions and a local renormalization group equation for general renormalizable field theories, Nucl. Phys. B 363 (1991) 486 [INSPIRE].

[30] A.B. Zamolodchikov, Irreversibility of the Flux of the Renormalization Group in a $2 D$ Field Theory, JETP Lett. 43 (1986) 730 [inSPIRE].

[31] I. Jack and H. Osborn, Analogs for the c Theorem for Four-dimensional Renormalizable Field Theories, Nucl. Phys. B 343 (1990) 647 [InSPIRE].

[32] J.L. Cardy, Is There a c Theorem in Four-Dimensions?, Phys. Lett. B 215 (1988) 749 [INSPIRE].

[33] B. Grinstein, A. Stergiou and D. Stone, Consequences of Weyl Consistency Conditions, JHEP 11 (2013) 195 [arXiv: 1308.1096] [INSPIRE].

[34] B. Grinstein, D. Stone, A. Stergiou and M. Zhong, Challenge to the a Theorem in Six Dimensions, Phys. Rev. Lett. 113 (2014) 231602 [arXiv:1406.3626] [INSPIRE].

[35] B. Grinstein, A. Stergiou, D. Stone and M. Zhong, Two-loop renormalization of multiflavor $\phi^{3}$ theory in six dimensions and the trace anomaly, Phys. Rev. D 92 (2015) 045013 [arXiv: 1504.05959] [INSPIRE].

[36] H. Osborn and A. Stergiou, Structures on the Conformal Manifold in Six Dimensional Theories, JHEP 04 (2015) 157 [arXiv:1501.01308] [INSPIRE].

[37] A. Stergiou, D. Stone and L.G. Vitale, Constraints on Perturbative RG Flows in Six Dimensions, JHEP 08 (2016) 010 [arXiv:1604.01782] [INSPIRE].

[38] I. Adam, I.V. Melnikov and S. Theisen, A Non-Relativistic Weyl Anomaly, JHEP 09 (2009) 130 [arXiv:0907.2156] [INSPIRE].

[39] P.R.S. Gomes and M. Gomes, On Ward Identities in Lifshitz-like Field Theories, Phys. Rev. D 85 (2012) 065010 [arXiv:1112.3887] [INSPIRE].

[40] J.-F. Fortin, B. Grinstein and A. Stergiou, Scale without Conformal Invariance: An Example, Phys. Lett. B 704 (2011) 74 [arXiv:1106.2540] [INSPIRE].

[41] J.-F. Fortin, B. Grinstein and A. Stergiou, Scale without Conformal Invariance: Theoretical Foundations, JHEP 07 (2012) 025 [arXiv: 1107.3840] [INSPIRE].

[42] J.-F. Fortin, B. Grinstein and A. Stergiou, Scale without Conformal Invariance at Three Loops, JHEP 08 (2012) 085 [arXiv: 1202.4757] [INSPIRE].

[43] J.-F. Fortin, B. Grinstein and A. Stergiou, Limit Cycles in Four Dimensions, JHEP 12 (2012) 112 [arXiv:1206.2921] [INSPIRE].

[44] J.-F. Fortin, B. Grinstein, C.W. Murphy and A. Stergiou, On Limit Cycles in Supersymmetric Theories, Phys. Lett. B 719 (2013) 170 [arXiv:1210.2718] [INSPIRE]. 
[45] J.-F. Fortin, B. Grinstein and A. Stergiou, Limit Cycles and Conformal Invariance, JHEP 01 (2013) 184 [arXiv:1208.3674] [InSPIRE].

[46] Z. Komargodski and A. Schwimmer, On Renormalization Group Flows in Four Dimensions, JHEP 12 (2011) 099 [arXiv: 1107.3987] [INSPIRE].

[47] M.A. Luty, J. Polchinski and R. Rattazzi, The a-theorem and the Asymptotics of $4 D$ Quantum Field Theory, JHEP 01 (2013) 152 [arXiv:1204.5221] [INSPIRE].

[48] V. Efimov, Energy levels arising form the resonant two-body forces in a three-body system, Phys. Lett. B 33 (1970) 563 [inSPIRE].

[49] L.H. Thomas, The Interaction Between a Neutron and a Proton and the Structure of $H^{3}$, Phys. Rev. 47 (1935) 903 [INSPIRE].

[50] D.D. Głazek and K.G. Wilson, Limit Cycles in Quantum Theories, Phys. Rev. Lett. (2002) 89230401.

[51] D.L. Jafferis, The Exact Superconformal R-Symmetry Extremizes Z, JHEP 05 (2012) 159 [arXiv: 1012.3210] [INSPIRE].

[52] D. Lovelock, The Einstein tensor and its generalizations, J. Math. Phys. 12 (1971) 498 [INSPIRE].

[53] R. Jackiw, Delta Function Potentials in Two and Three Dimensions, in M.A.B. Beg Memorial Volume, World Scientific, Singapore (1991), p. 25.

[54] Y. Nishida and D.T. Son, Nonrelativistic conformal field theories, Phys. Rev. D 76 (2007) 086004 [arXiv: 0706 . 3746] [INSPIRE].

[55] R. Jackiw, Dynamical Symmetry of the Magnetic Monopole, Annals Phys. 129 (1980) 183 [INSPIRE].

[56] R. Jackiw, Dynamical Symmetry of the Magnetic Vortex, Annals Phys. 201 (1990) 83 [INSPIRE].

[57] R. Britto-Pacumio, J. Michelson, A. Strominger and A. Volovich, Lectures on Superconformal Quantum Mechanics and Multi-Black Hole Moduli Spaces, NATO Sci. Ser. C 556 (2000) 255 [hep-th/9911066] [INSPIRE].

[58] C. Chamon, R. Jackiw, S.-Y. Pi and L. Santos, Conformal quantum mechanics as the CFT dual to $A d S_{2}$, Phys. Lett. B 701 (2011) 503 [arXiv:1106.0726] [INSPIRE].

[59] R. Jackiw and S.Y. Pi, Conformal Blocks for the 4-Point Function in Conformal Quantum Mechanics, Phys. Rev. D 86 (2012) 045017 [Erratum ibid. D 86 (2012) 089905] [arXiv: 1205.0443] [INSPIRE].

[60] V. de Alfaro, S. Fubini and G. Furlan, Conformal Invariance in Quantum Mechanics, Nuovo Cim. 34A (1976) 569.

[61] A. Sen, State Operator Correspondence and Entanglement in $A d S_{2} / C F T_{1}$, Entropy 13 (2011) 1305 [arXiv: 1101.4254] [INSPIRE].

[62] K.M. Case, Singular potentials, Phys. Rev. 80 (1950) 797 [INSPIRE].

[63] K.S. Gupta and S.G. Rajeev, Renormalization in quantum mechanics, Phys. Rev. D 48 (1993) 5940 [hep-th/9305052] [INSPIRE].

[64] H.E. Camblong, L.N. Epele, H. Fanchiotti and C.A. Garcia Canal, Renormalization of the inverse square potential, Phys. Rev. Lett. 85 (2000) 1590 [hep-th/0003014] [INSPIRE]. 
[65] B. Basu-Mallick, P.K. Ghosh and K.S. Gupta, Novel quantum states of the rational Calogero models without the confining interaction, Nucl. Phys. B 659 (2003) 437 [hep-th/0207040] [INSPIRE].

[66] A.M. Essin and D.J. Griffiths, Quantum mechanics of the $1 / x^{2}$ potential, Am. J. Phys. 74 (2005) 109.

[67] H.W. Hammer and B.G. Swingle, On the limit cycle for the $1 / R^{2}$ potential in momentum space, Annals Phys. 321 (2006) 306 [quant-ph/0503074] [INSPIRE].

[68] S.R. Beane, P.F. Bedaque, L. Childress, A. Kryjevski, J. McGuire and U. van Kolck, Singular potentials and limit cycles, Phys. Rev. A 64 (2001) 042103 [quant-ph/0010073] [INSPIRE].

[69] P.F. Bedaque, H.W. Hammer and U. van Kolck, Renormalization of the three-body system with short range interactions, Phys. Rev. Lett. 82 (1999) 463 [nucl-th/9809025] [INSPIRE].

[70] H.E. Camblong, L.N. Epele, H. Fanchiotti and C.A. Garcia Canal, Quantum anomaly in molecular physics, Phys. Rev. Lett. 87 (2001) 220402 [hep-th/0106144] [INSPIRE].

[71] S. Pal and N. Banerjee, Addressing the issue of nonunitarity in anisotropic quantum cosmology, Phys. Rev. D 90 (2014) 104001 [arXiv:1410.2718] [INSPIRE].

[72] S. Pal and N. Banerjee, Restoring unitarity in anisotropic quantum cosmological models, Phys. Rev. D 91 (2015) 044042 [arXiv:1411.1167] [INSPIRE].

[73] S. Pal, Physical Aspects of Unitary evolution of Bianchi-I Quantum Cosmological Model, Class. Quant. Grav. 33 (2016) 045007 [arXiv: 1504.02912] [INSPIRE].

[74] A. Strominger, Black hole entropy from near horizon microstates, JHEP 02 (1998) 009 [hep-th/9712251] [INSPIRE]. 